\title{
The Myopic Stable Set for Social Environments
}

Citation for published version (APA):

Demuynck, T., Herings, P. J-J., Saulle, R., \& Seel, C. (2017). The Myopic Stable Set for Social

Environments. Maastricht University, Graduate School of Business and Economics. GSBE Research Memoranda No. 002 https://doi.org/10.26481/umagsb.2017002

Document status and date:

Published: 01/01/2017

DOI:

10.26481/umagsb.2017002

Document Version:

Publisher's PDF, also known as Version of record

\section{Please check the document version of this publication:}

- A submitted manuscript is the version of the article upon submission and before peer-review. There can be important differences between the submitted version and the official published version of record.

People interested in the research are advised to contact the author for the final version of the publication, or visit the DOI to the publisher's website.

- The final author version and the galley proof are versions of the publication after peer review.

- The final published version features the final layout of the paper including the volume, issue and page numbers.

Link to publication

\footnotetext{
General rights rights.

- You may freely distribute the URL identifying the publication in the public portal. please follow below link for the End User Agreement:

www.umlib.nl/taverne-license

Take down policy

If you believe that this document breaches copyright please contact us at:

repository@maastrichtuniversity.nl

providing details and we will investigate your claim.
}

Copyright and moral rights for the publications made accessible in the public portal are retained by the authors and/or other copyright owners and it is a condition of accessing publications that users recognise and abide by the legal requirements associated with these

- Users may download and print one copy of any publication from the public portal for the purpose of private study or research.

- You may not further distribute the material or use it for any profit-making activity or commercial gain

If the publication is distributed under the terms of Article $25 \mathrm{fa}$ of the Dutch Copyright Act, indicated by the "Taverne" license above, 


\section{Maastricht University}

Thomas Demuynck,

P. Jean-Jacques Herings,

Riccardo D. Saulle, Christian Seel

The Myopic Stable Set for Social Environments

$\mathrm{RM} / 17 / 002$

\section{GSBE}

Maastricht University School of Business and Economics

Graduate School of Business and Economics

P.O Box 616

NL- 6200 MD Maastricht

The Netherlands 


\title{
The Myopic Stable Set for Social Environments
}

\author{
Thomas Demuynck $^{*} \quad$ P. Jean-Jacques Herings ${ }^{\dagger} \quad$ Riccardo D. Saulle ${ }^{\ddagger}$ \\ Christian Seel $\S$
}

December 21, 2016

\begin{abstract}
We introduce a new solution concept for models of coalition formation, called the myopic stable set. The myopic stable set is defined for a very general class of social environments and allows for an infinite state space. We show that the myopic stable set exists and is non-empty. Under minor continuity conditions, we also demonstrate uniqueness. Furthermore, the myopic stable set is a superset of the core and of the set of pure strategy Nash equilibria in noncooperative games.

Additionally, the myopic stable set generalizes and unifies various results from more specific environments. In particular, the myopic stable set coincides with the coalition structure core in coalition function form games if the coalition structure core is nonempty; with the set of stable matchings in the standard one-to-one matching model; with the set of pairwise stable networks and closed cycles in models of network formation; and with the set of pure strategy Nash equilibria in finite supermodular games, finite potential games, and aggregative games. We illustrate the versatility of our concept by characterizing the myopic stable set in a model of Bertrand competition with asymmetric costs, for which the literature so far has not been able to fully characterize the set of all (mixed) Nash equilibria.
\end{abstract}

KEYWORDS: Social environments, group formation, stability, Nash equilibrium. JEL classification: $C 70, C 71$.

\footnotetext{
*Ecares, Université Libre de Bruxelles, Belgium. E-Mail: Thomas.Demuynck@ulb.ac.be

${ }^{\dagger}$ Department of Economics, Maastricht University, Maastricht, The Netherlands. E-Mail: P.Herings@maastrichtuniversity.nl

${ }_{\ddagger}^{\ddagger}$ Department of Economics, Maastricht University, Maastricht, The Netherlands. E-Mail: R.Saulle@maastrichtuniversity.nl

$\S$ Department of Economics, Maastricht University, Maastricht, The Netherlands. E-Mail: C.Seel@maastrichtuniversity.nl
} 


\section{Introduction}

Models of coalition formation study a widespread and important pattern of human interaction: agents tend to form groups of equally interested individuals, but these groups behave in a non-cooperative way towards outsiders. For example, individuals in a community join forces to provide a local public good, voters create parties to attain their political goals, and firms set up lobby groups to influence policy-makers.

The literature studies coalition formation in many distinct settings, like networks, coalition function games, and matching models. In this paper, we focus on a general class of social environments that covers all of these settings and many more. More precisely, we define a social environment on the basis of four components (Chwe, 1994): a finite collection of agents, a set of social states, for each agent, preferences over the set of states and an effectivity correspondence that models the feasible transitions from one state to another. We only require that the set of social states is a non-empty and compact metric space. As such, in contrast to most settings in the literature, we allow the state space to be infinite.

For such social environments, we define a new solution concept called the myopic stable set, abbreviated as MSS. The MSS extends the idea of level-1 farsighted stability by Herings, Mauleon, and Vannetelbosch $(2009,2014)$ from finite networks to the general class of social environments. The MSS is defined by three conditions, deterrence of external deviations, external stability and minimality. Deterrence of external deviations requires that there is no coalition that benefits from a deviation from a state inside the MSS to a state outside the MSS. External stability makes sure that from any state outside the set it is possible to get arbitrarily close to a state inside the MSS by a sequence of coalitional deviations. The final condition, minimality, requires that the MSS is minimal with respect to set inclusion.

Our notion of dominance is myopic in the sense that agents (or coalitions) do not predict how their decision to change the current state to another one will lead to further changes by other coalitions. Such a notion is natural in very complex social environments where the number of possible states and possible actions is overwhelmingly large and agents have little information about the possible actions other agents may take or the incentives of other agents. The myopic stable set thereby distinguishes our approach from the ones in the literature that focus on farsightedness (see among others, Chwe, 1994; Xue, 1998; Herings, Mauleon, and Vannetelbosch, 2004, 2009, 2014; Dutta, Ghosal, and Ray, 2005; Page, Wooders, and Kamat, 2005; Page and Wooders, 2009; Ray and Vohra, 2015). On the other hand, our analysis is more in line with myopic concepts like the core and the von Neumann-Morgenstern stable set. As we will see in the application to normal-form games, it is also intimately connected to the notion of Nash equilibrium.

Our first main result (Theorem 4.1) shows that every social environment contains at least one non-empty MSS. Moreover, under weak continuity assumptions we establish uniqueness 
of the MSS (Theorem 4.6). The existence and uniqueness results differ from many other popular solution concepts in the literature. For instance, the core and the coalition structure core for coalition function form games can be empty (Bondareva, 1963; Scarf, 1967; Shapley, 1967); the von Neumann Morgenstern stable set may fail to exist or to be unique (Lucas, 1968, 1992) and the set of pure strategy Nash equilibria may be empty.

We also provide several additional results that provide more insights about the structure of an MSS (Theorem 4.12). For finite state spaces, we fully characterize the MSS as the union of all closed cycles, i.e., subsets which are closed under coalitional better replies. For infinite spaces, the union of all closed cycles is found to be a subset of the MSS. This result is helpful in applications and in the comparison to other solution concepts. For instance, any state in the core is a closed cycle and is therefore included in the MSS. Next we define a generalization of the weak improvement property (Friedman and Mezzetti, 2001) to social environments and we show that, under weak continuity conditions, the weak improvement property characterizes the collection of social environments for which the MSS coincides with the core.

We demonstrate the versatility of these results by analyzing the relationship between the MSS and other solution concepts in more specific social environments. In particular, we show that the MSS coincides with the coalition structure core for coalition function form games (Kóczy and Lauwers, 2004) whenever the coalition structure core is non-empty; with the set of stable matchings in the one-to-one matching model by Gale and Shapley (1962); with the set of the set of pairwise stable networks and closed cycles in models of network formation (Jackson and Watts, 2002), and the set of pure strategy Nash equilibria in finite supermodular games (Bulow, Geanakoplos, and Klemperer, 1985), finite potential games (Monderer and Shapley, 1996), and aggregative games (Selten, 1970). Finally, we illustrate the versatility of our results by characterizing the MSS in a model of Bertrand competition with asymmetric costs. This model is characterized by discontinuous payoff functions and has no pure-strategy Nash equilibrium. Although Blume (2003) has shown the existence of a mixed-strategy Nash equilibrium, the literature has, so far, not been able to characterize the complete class of (mixed) equilibria for this game.

The structure of the paper is as follows. Section 2 provides the primitives of our general framework of social environments and introduces several applications to demonstrate its generality. Section 3 introduces and motivates the MSS. Section 4 establishes existence, non-emptiness, and uniqueness results. Section 5 analyzes our solution concept for the examples provided in Section 2 and relates it to other stability concepts from the literature. Finally, Section 6 concludes. 


\section{General Framework}

Let $N$ be a non-empty finite set of individuals with cardinality $n$. A coalition $S$ is a subset of $N$. The set of non-empty subsets of $N$ is denoted by $\mathcal{N}$. Let $(X, d)$ be a metric space, where $X$ denotes our non-empty state space and $d$ is a metric on $X .{ }^{1}$ Let some state $x \in X$ be given. We define

$$
B_{\varepsilon}(x)=\{y \in X \mid d(x, y)<\varepsilon\}
$$

as the open ball around $x$ with radius $\varepsilon$. The set $B_{\varepsilon}(x)$ contains all the states in $X$ that are in an $\varepsilon$-neighborhood of $x$. An effectivity correspondence $E$ associates with each pair of states $x, y \in X$ a, possibly empty, collection of coalitions $E(x, y) \subseteq \mathcal{N}$. If $S \in E(x, y)$, we say that the coalition $S$ can move from state $x$ to state $y$. If $E(x, y)=\emptyset$, then no coalition can move from $x$ to $y$.

Each individual $i \in N$ has a complete and transitive preference relation $\succeq_{i}$ over the state space $X$. The profile $\left(\succeq_{i}\right)_{i \in N}$ then lists the preferences of all individuals in $N$. We denote by $\succ_{i}$ the asymmetric part of $\succeq_{i}$, i.e., $x \succ_{i} y$ if and only if $x \succeq_{i} y$ and not $y \succeq_{i} x$.

A social environment is now defined as follows.

Definition 2.1 (Social Environment). A social environment is a tuple

$$
\Gamma=\left(N,(X, d), E,\left(\succeq_{i}\right)_{i \in N}\right)
$$

consisting of a non-empty, finite set of agents $N$, a non-empty, compact metric space $(X, d)$ of states, an effectivity correspondence $E$ on $X$, and a collection of preference relations $\left(\succeq_{i}\right)_{i \in N}$ over $X$.

The state space $(X, d)$ can be used to encode many aspects of a particular application. To illustrate the generality of our setting, we provide four specific models that have been studied extensively in the literature: coalition function form games, one-to-one matching models, models of network formation, and non-cooperative normal-form games. For each of these examples we specify the social environment, i.e., the set of players $N$, the state space $(X, d)$, the preferences $\left(\succeq_{i}\right)_{i \in N}$, and the effectivity correspondence $E$.

\subsection{Coalition Function Form Games}

A coalition function form game is defined by a tuple $(N, v)$, where $N$ is the set of players and $v: 2^{N} \rightarrow \mathbb{R}$ is a characteristic function that assigns to each coalition $S \subseteq N$ a number

\footnotetext{
${ }^{1} \mathrm{~A}$ metric $d$ is a function $d: X \times X \rightarrow \mathbb{R}_{+}$such that (i) for every $x, y \in X: d(x, y)=0$ if and only if $x=y$, (ii) for every $x, y \in X: d(x, y)=d(y, x)$, and (iii) for every $x, y, z \in X, d(x, y) \leq d(x, z)+d(z, y)$.
} 
$v(S) \in \mathbb{R}$, called the coalitional value of $S$, with the usual convention that $v(\emptyset)=0$. A coalition structure is a partition $\pi:=\left\{S^{1}, \ldots, S^{m}\right\}$ of $N$. It describes how the grand coalition is divided into various sub-coalitions. The collection of all coalition structures, i.e., the collection of partitions of $N$, is denoted by $\Pi$.

For coalition function form games, we define $X$ as the set of coalition structures $\Pi$ together with all individually rational payoff vectors that can be obtained by allocating the coalitional values among the members of the respective coalitions:

$$
X=\left\{(\pi, u) \in \Pi \times \mathbb{R}^{N} \mid \forall i \in N: u_{i} \geq v(\{i\}) \text { and } \forall S \in \pi: \sum_{i \in S} u_{i}=v(S)\right\} .
$$

Given a state $x \in X$, we denote by $\pi(x)$ the projection to its first component, i.e., the coalition structure, and by $u(x)$ the projection to its second component, i.e., the payoff vector, so we can write $x=(\pi(x), u(x))$. The restriction of the payoff vector $u(x)$ to the members in coalition $S$ is denoted by $u_{S}(x)$. The set $X$ is non-empty since it always contains the state where $N$ is partitioned into singletons and each player $i \in N$ receives the payoff $v(i)$.

We define the metric $d$ on $X$ by setting for all $x, y \in X$,

$$
d(x, y)=\mathbb{1}_{\{\pi(x) \neq \pi(y)\}}+\|u(x)-u(y)\|_{\infty},
$$

where $\mathbb{1}$ is the indicator function and $\|\cdot\|_{\infty}$ is the infinity norm.

We define preferences $\succeq_{i}$ over the state space $X$ by setting $x \succeq_{i} y$ if and only if $u_{i}(x) \geq$ $u_{i}(y)$, i.e., the payoff for individual $i$ in state $x$ is at least as high as the payoff for individual $i$ in state $y$.

For each pair of states $(x, y)$, the effectivity correspondence $E(x, y)$ specifies which coalitions can change state $x$ into state $y$. As an example that imposes some reasonable structure on the effectivity correspondence, we provide a brief outline of the restriction of coalitional sovereignty (Konishi and Ray, 2003; Kóczy and Lauwers, 2004; Ray and Vohra, 2014, 2015; Herings, Mauleon, and Vannetelbosch, 2016). For coalitional sovereignty, a group of players $S$ - the leaving players - can decide to leave their partners and create a new group. This event induces a change from a state $x$ to a state $y$ characterized by a new coalition structure and a new payoff vector. The collection of coalitions that is unaffected by this change is denoted $\mathcal{U}(x, S)$ and the set of players that is unaffected is denoted by $U(x, S)$. The set $\mathcal{U}(x, S)$ contains all coalitions $T \in \pi(x)$ that are disjoint from $S$. Formally,

$$
\begin{aligned}
& \mathcal{U}(x, S)=\{T \in \pi(x) \mid S \cap T=\emptyset\}, \\
& U(x, S)=\cup_{T \in \mathcal{U}(x, S) T .}
\end{aligned}
$$

An effectivity correspondence is in accordance with the principle of coalitional sovereignty if it satisfies the properties of Non-interference and Full support. Non-interference requires 
that if a coalition $S$ changes from a state $x$ to a state $y$, then the unaffected coalitions $\mathcal{U}(x, S)$ are still part of the new coalition structure $\pi(y)$ and every unaffected individual $i \in U(x, S)$ keeps her old payoff, i.e., $u_{i}(x)=u_{i}(y)$.

(1) Non-interference: For every $x, y \in X$, if $S \in E(x, y)$ and $T \in \mathcal{U}(x, S)$, then $S \in$ $\pi(y), T \in \pi(y)$, and $u_{T}(x)=u_{T}(y)$

Full support requires that every coalition $S$ has the opportunity to move to a new state where it has the freedom to redistribute its worth $v(S)$ at will.

(2) Full support: For every $x \in X$, every $S \in \mathcal{N}$, and every $u \in \mathbb{R}^{S}$ such that for all $i \in S: u_{i} \geq v(\{i\})$ and $\sum_{i \in S} u_{i}=v(S)$, there is a state $y \in X$ such that $u_{S}(y)=u$ and $S \in E(x, y)$.

Coalitional sovereignty does not fully specify the effectivity correspondence $E$. In particular, it leaves unspecified the payoffs and coalition structure of players that are neither part of the leaving coalition $S$ nor part of an unaffected collection $U(x, S)$, i.e., players in the set $N \backslash(S \cup U(x, S))$. We call these players residual players. Indeed, one of the more controversial issues is to what extent the leaving players have the power to influence the coalition structure and payoffs of these residual players; see Shubik (1962), Hart and Kurz (1983), Konishi and Ray (2003), and Ray and Vohra (2014) for related discussions and alternative viewpoints. One frequently used specification is the $\gamma$-model (Hart and Kurz, 1983). This $\gamma$-model prescribes that the residual players are divided into singletons. This assumption is justified by the viewpoint that a coalition is only maintained if there is unanimous agreement among its members. As such, the departure of one individual implies the collapse of the entire coalition. In our setting, the $\gamma$-model imposes the following restriction on the effectivity correspondence.

(3) $\boldsymbol{\gamma}$-model For all $x, y \in X$ and $S \in E(x, y)$, if $i \in N \backslash(S \cup U(x, S))$, then $\{i\} \in \pi(y)$.

\subsection{One-to-One Matching}

As a second example of a social environment, we consider the two sided one-to-one matching model $\left(M, W,\left(P_{m}\right)_{m \in M},\left(P_{w}\right)_{w \in W}\right)$ from Gale and Shapley (1962). The matching model consists of a finite set $N$ of individuals, partitioned in the two exhaustive subgroups of men $M$ and women $W$. A matching is a function $\mu: M \cup W \rightarrow M \cup W$ satisfying the following properties:

1. For every man $m \in M, \mu(m) \in W \cup\{m\}$.

2. For every women $w \in W, \mu(w) \in M \cup\{w\}$. 
3. For all men $m \in M$ and women $w \in W, \mu(m)=w$ if and only if $\mu(w)=m$.

In this setting, our state space $X$ consists of all possible matchings $\mu$. Since $X$ is finite, we can endow it with the discrete metric

$$
d\left(\mu, \mu^{\prime}\right)=\mathbb{1}_{\left\{\mu \neq \mu^{\prime}\right\}} .
$$

Each man $m \in M$ has a complete and transitive strict preference relation $P_{m}$ over the set $W \cup\{m\}$ and each woman $w \in W$ has a complete and transitive strict preference relation $P_{w}$ over the set $M \cup\{w\}$. We assume that the preferences of the individuals $\left(\succeq_{i}\right)_{i \in M \cup W}$ over the set $X$ are induced by their preferences over their match, i.e., for all $m \in M$ it holds that $\mu \succ_{m} \mu^{\prime}$ if and only if $\mu(m) P_{m} \mu^{\prime}(m)$ and for all $w \in W$ it holds that $\mu \succ_{w} \mu^{\prime}$ if and only if $\mu(w) P_{w} \mu^{\prime}(w)$.

Imposing restrictions on the effectivity correspondence allows us to study the consequences of different hypothesis on the matching process. We introduce two common restrictions from the literature on matching. The first requires that every man or woman is allowed to break his/her link with her/his current partner. Doing so makes this man or women and their former partners single.

(1) For all $i \in N$ and $\mu \in X$ with $\mu(i) \neq i$, we have $\{i\} \in E\left(\mu, \mu^{\prime}\right)$ where $\mu^{\prime} \in X$ is such that

(i) $\mu^{\prime}(i)=i$,

(ii) $\mu^{\prime}(\mu(i))=\mu(i)$, and

(iii) for every $j \in N \backslash\{i, \mu(i)\}$ we have $\mu^{\prime}(j)=\mu(j)$.

The second restriction requires than any man and woman that are currently not matched to each other can deviate by creating a link and thereby leaving their former partners single.

(2) For all $m^{\prime} \in M, w^{\prime} \in W$, and $\mu \in X$ with $\mu\left(m^{\prime}\right) \neq w^{\prime}$, we have that $\left\{m^{\prime}, w^{\prime}\right\} \in E\left(\mu, \mu^{\prime}\right)$, where $\mu^{\prime} \in X$ is such that

(i) $\mu^{\prime}\left(m^{\prime}\right)=w^{\prime}$

(ii) $\mu\left(m^{\prime}\right) \in W$ implies $\mu^{\prime}\left(\mu\left(m^{\prime}\right)\right)=\mu\left(m^{\prime}\right)$

(iii) $\mu\left(w^{\prime}\right) \in M$ implies $\mu^{\prime}\left(\mu\left(w^{\prime}\right)\right)=\mu\left(w^{\prime}\right)$,

(iv) for every $j \in N \backslash\left\{m^{\prime}, w^{\prime}, \mu\left(m^{\prime}\right), \mu\left(w^{\prime}\right)\right\}$ it holds that $\mu^{\prime}(j)=\mu(j)$.

Observe that these two conditions respect the $\gamma$-model of coalitional sovereignty. 


\subsection{Network Formation}

As a third example of a social environment, we look at models of network formation (Jackson and Wolinsky, 1996). A network is given by a tuple $g=(N, \mathcal{E})$, where $N$ are the nodes/players of the network and $\mathcal{E}$ is the set of undirected edges of the network. An undirected edge is represented as a set of two distinct nodes/players. Two players $i, j \in N$ are linked in $g$ if and only if $\{i, j\} \in \mathcal{E}$. We often abuse notation and write $i j \in g$ if $i$ and $j$ are linked in the network $g$. The set of all networks with node set $N$ is denoted by $\mathbb{G}$. A value function for player $i$ is a a function $v_{i}: \mathbb{G} \rightarrow \mathbb{R}$ that associates a payoffs for player $i$ for each network in $\mathbb{G}$. A network problem is thus given by $\left(N, \mathbb{G},\left(v_{i}\right)_{i \in N}\right)$.

We identify $X$ with the set $\mathbb{G}$ of all possible networks on $N$ and endow it with the discrete metric

$$
d\left(g, g^{\prime}\right)=\mathbb{1}_{\left\{g \neq g^{\prime}\right\}}
$$

Every agent $i \in N$ has a preference relation $\succeq_{i}$ over the set $X$ of all possible networks defined by $g \succeq_{i} g^{\prime}$ if $v_{i}(g) \geq v_{i}\left(g^{\prime}\right)$. Let $g+i j$ be the network obtained from network $g$ by adding the link $i j$ and let $g-i j$ be the network obtained by deleting the link $i j$ from $g$.

We follow Jackson and Wolinsky (1996) by considering deviations by coalitions of size one or two and by assuming link-deletion to be one-sided and link addition to be two-sided. One-sided link deletion allows every player to delete one of its links.

(1) For all individuals $i \in N$, all networks $g \in X$, and all links $i j \in g,\{i\} \in E(g, g-i j)$.

Two sided link addition allows any two players that are currently not-linked can change the network by forming a link between themselves.

(2) For all individuals $i, j \in N$, all networks $g \in X$ with $i j \notin g$, we have $\{i, j\} \in E(g, g+i j)$.

It is straightforward to adjust the effectivity correspondence to incorporate models of network formation where more than one link at a time can be changed by coalitions of arbitrary size (Dutta and Mutuswami, 1997; Jackson and van den Nouweland, 2005) or where link formation is one-sided (Bala and Goyal, 2000) into our framework. We refer to Page and Wooders (2009) for a more extensive discussion of alternative rules of network formation.

\subsection{Normal-Form Games}

As a last example of a social environment, we take non-cooperative normal-form games. A normal-form game is given by a triple $G=\left(N,\left(\Sigma_{i}, d_{i}\right)_{i \in N},\left(\succeq_{i}\right)_{i \in N}\right)$, where $N$ is the set of players, $\Sigma_{i}$ is the set of strategies for player $i$ modelled as a metric space with metric $d_{i}$, and $\succeq_{i}$ is a preference relation over the set of all strategy profiles $\Sigma=\prod_{i \in N} \Sigma_{i}$ with typical 
element $\sigma$. We assume that each strategy set $\left(\Sigma_{i}, d_{i}\right)$ is non-empty and compact. We identify the state space $X$ with the set of strategy profiles $\Sigma$ and endow it with the product metric

$$
d\left(\sigma, \sigma^{\prime}\right)=\sum_{i=1}^{n} d_{i}\left(\sigma_{i}, \sigma_{i}^{\prime}\right)
$$

As is standard in non-cooperative game theory, we use the notation $\left(\sigma_{i}, \sigma_{-i}\right)$ for the strategy profile where $\sigma_{i}$ is the strategy of player $i$ and $\sigma_{-i}$ is the list of strategies of all players except $i$, i.e., $\sigma_{-i}:=\left(\sigma_{j}\right)_{j \in N \backslash\{i\}}$.

To define the effectivity correspondence, first note that the standard analysis of a noncooperative game only allows coalitions of size one. Only singletons are effective and can change the state by a unilateral deviation from their strategy. This means that for every player $i \in N$, coalition $\{i\}$ can change the state $\sigma=\left(\sigma_{i}, \sigma_{-i}\right)$ to the state $\sigma^{\prime}$, i.e., $\{i\} \in$ $E\left(\sigma, \sigma^{\prime}\right)$ if and only if $\sigma^{\prime}=\left(\sigma_{i}^{\prime}, \sigma_{-i}\right)$ for some $\sigma_{i}^{\prime} \in \Sigma_{i}$.

In this example we stick to the standard interpretation of a normal-form game where only individuals can deviate. By adjusting the effectivity correspondence, our framework can easily accommodate deviations by groups of players as for instance considered in the concept of strong Nash equilibrium introduced in Aumann (1959).

\section{The Solution Concept}

In this section, we first introduce the notions of dominance and asymptotic dominance which are used to define our solution concept, the myopic stable set.

We say that a state $y \in X$ dominates another state $x \in X$ if there is a coalition which can (i) move from $x$ to $y$ and (ii) each member of the coalition that makes the move strictly prefers $y$ over $x$.

Definition 3.1 (Dominance). A state $y \in X$ dominates the state $x \in X$ under $E$ if there exists a coalition $S \in E(x, y)$ such that for every $i \in S$ it holds that $y \succ_{i} x$.

Let some state $x \in X$ be given. The subset of $X$ consisting of all states that dominate $x$ together with state $x$ itself is denoted by $f(x)$, so

$$
f(x)=\{x\} \cup\{y \in X \mid y \text { dominates } x \text { under } E\} .
$$

We define the two-fold composition of $f$ by

$$
f^{2}(x)=\{z \in X \mid \exists y \in S: y \in f(x) \text { and } z \in f(y)\} .
$$

By induction, we can define the $k$-fold iteration $f^{k}(x)$ as the subset of $X$ that contains all states obtained by a composition of dominance correspondences of length $k \in \mathbb{N}$, i.e., 
$y \in f^{k}(x)$ if there is a $z \in X$ such that $y \in f(z)$ and $z \in f^{k-1}(x)$. Observe that for all $k, \ell \in \mathbb{N}$ if $k \leq \ell$, then $f^{k}(x) \subseteq f^{\ell}(x)$. We define the set of all states that can be reached from $x$ by a finite number of dominations by $f^{\mathbb{N}}(x)$, so

$$
f^{\mathbb{N}}(x):=\bigcup_{k \in \mathbb{N}} f^{k}(x)
$$

A state $y$ is said to asymptotically dominate the state $x$ if starting from $x$, it is possible to get arbitrarily close to $y$ in a finite number of dominations.

Definition 3.2 (Asymptotic Dominance). A state $y \in X$ asymptotically dominates the state $x \in X$ under $E$ if for all $\varepsilon>0$ there exists a number $k \in \mathbb{N}$ and a state $z \in f^{k}(x)$ such that $d(y, z)<\varepsilon$.

We denote by $f^{\infty}(x)$ the set of all states in $X$ that asymptotically dominate $x$. Formally,

$$
f^{\infty}(x)=\left\{y \in X \mid \forall \varepsilon>0, \exists k \in \mathbb{N}, \exists z \in f^{k}(x): d(y, z)<\varepsilon\right\} .
$$

It is easy to see that the set $f^{\infty}(x)$ coincides with the closure of the set $f^{\mathbb{N}}(x)$.

We are now ready to define our solution concept, the myopic stable set, abbreviated as MSS.

Definition 3.3 (Myopic Stable Set). Let $\Gamma=\left(N,(X, d), E,\left(\succeq_{i}\right)_{i \in N}\right)$ be a social environment. The set $M \subseteq X$ is a myopic stable set if it is closed and satisfies the following three conditions:

1. Deterrence of external deviations: For all $x \in M, f(x) \subseteq M$.

2. External stability: For all $x \notin M, f^{\infty}(x) \cap M \neq \emptyset$.

3. Minimality: There is no closed set $M^{\prime} \subsetneq M$ that satisfies Conditions 1 and 2 .

Let $M$ be a MSS. Deterrence of external deviations requires that no coalition can profitably deviate to a state outside $M$. External stability requires that any state outside $M$ is asymptotically dominated by a state in $M$. Hence, from any state outside of $M$ it is possible to get arbitrary close to a state in $M$ by a finite number of dominations. Observe that an empty set would necessarily violate external stability, so any MSS is non-empty. Minimality imposes that there is no smaller closed set of states that satisfies deterrence of external deviations and external stability.

For finite state spaces, it does not matter if one uses $f^{\mathbb{N}}$ or $f^{\infty}$ in the definition of external stability. On the other hand, for infinite state spaces, the asymptotic dominance relation $f^{\infty}$ is the natural extension of $f^{\mathbb{N}}$. Also when the state space is infinite, an MSS might fail to exist if one uses $f^{\mathbb{N}}$ instead of $f^{\infty}$ in the definition of external stability. This is illustrated in the following example. 
Example 1. Consider the social environment

$$
\Gamma=\left(\{1\},(X, d), \succeq_{1}\right)
$$

where,

$$
X=\left\{\frac{1}{k} \mid k \in \mathbb{N}\right\} \cup\{0\},
$$

and $d$ is the usual metric on $X, d(x, y)=|x-y|$. As such, $X$ is closed and compact. Preferences $\succeq_{1}$ are defined by $x \succeq_{1} y$ if and only if $x=y$ or $y>x \geq 0$. The effectivity correspondence $E$ is such that $\{1\} \in E(1 / k, 1 /(k+1))$ for every $k \in \mathbb{N}$ and $E(x, y)=\emptyset$ otherwise. It follows that

$$
f(1 / k)=\{1 / k, 1 /(k+1)\} .
$$

Observe that $0 \in f^{\infty}(x)$ for every $x \in X$ and that $f(0)=\{0\}$. It now follows easily that $\{0\}$ is an MSS.

Suppose we replace the requirement of external stability by the stronger notion that for all states $x \notin M, f^{\mathbb{N}}(x) \cap M \neq \emptyset$, a property that we refer to as strong external stability. Since, for every $k \in \mathbb{N}, 0 \notin f^{\mathbb{N}}(1 / k)$, the set $\{0\}$ does not satisfy strong external stability. Actually, we can show that there is no closed set satisfying strong external stability together with deterrence of external deviations and minimality. Towards a contradiction, assume that the closed set $M \subseteq X$ satisfies these properties. Given that $M \neq\{0\}$ and $M$ is non-empty, there is $k \in \mathbb{N}$ such that $1 / k \in M$. Moreover, let $k$ be the smallest such number. It is easy to verify that the closed set $M^{\prime}:=M \backslash\{1 / k\}$ satisfies deterrence of external deviations and strong external stability. Since $M^{\prime}$ is a proper subset of $M, M$ violates the minimality property.

An MSS is defined as a minimal set satisfying deterrence of external deviations and external stability. Dropping the minimality requirement, we can define the concept of a quasi myopic stable set (QMSS) which is useful in the proofs.

Definition 3.4 (Quasi Myopic Stable Set). Let $\Gamma$ be a social environment. The set $M \subseteq X$ is a quasi myopic stable set if it is closed and satisfies deterrence of external deviations and external stability.

\section{General Properties}

This section establishes existence of the myopic stable set in general and, under weak additional assumptions, its uniqueness. The final part of this section derives some additional structural properties of myopic stable sets that are used in the next section. 


\subsection{Existence}

The following main result shows existence of the myopic stable set.

Theorem 4.1 (Existence). Let $\Gamma$ be a social environment. Then an MSS exists.

Proof. First observe that the set of states $X$ is a QMSS. Indeed, since it is compact, it is closed and it trivially satisfies deterrence of external deviations and external stability.

Let $\mathcal{Z}$ be the collection of all sets of states that are a QMSS. Notice $\mathcal{Z}$ is non-empty as $X \in \mathcal{Z}$. We will use Zorn's lemma to show the existence of a minimal element in the partially ordered set $(\mathcal{Z}, \supseteq)$, i.e., a set $Z \in \mathcal{Z}$ is minimal element if for all $Z^{\prime} \in \mathcal{Z}$ with $Z^{\prime} \subseteq Z$, we have $Z=Z^{\prime}$.

Let $\mathcal{S}$ be a chain in $\mathcal{Z}$, i.e., $(\mathcal{S}, \supseteq)$ is a totally ordered subset of $(\mathcal{Z}, \supseteq)$. Let $I$ be an index set for the sets in $\mathcal{S}$, i.e., $\mathcal{S}=\left\{Z^{\alpha} \mid \alpha \in I\right\}$. Let $\triangleright$ be the order on $I$ that is induced by the order on $\mathcal{S}$, i.e., $\alpha \triangleright \beta$ if and only if $Z^{\alpha} \subseteq Z^{\beta}$. In order to apply Zorn's Lemma, we have to show that $\mathcal{S}$ has a lower bound in $\mathcal{Z}$. Let $M=\bigcap_{\alpha \in I} Z^{\alpha}$. Clearly, $M$ is a lower bound of $\mathcal{S}$. We proceed by showing that $M \in \mathcal{Z}$, i.e., $M$ is a QMSS. First of all, observe that $M$ is closed as it is defined as the intersection of a collection of closed sets. We need to show that it satisfies deterrence of external deviations and external stability.

Deterrence of external deviations: Let $x \in M$ and $y \notin M$ be given. Then there is $\alpha \in I$ such that $y \notin Z^{\alpha}$, since otherwise $y \in Z^{\alpha}$ for all $\alpha \in I$, which means that $y \in M$. Since $x \in Z^{\alpha}$ and $Z^{\alpha}$ satisfies deterrence of external deviations, we obtain $y \notin f(x)$ as was to be shown.

External stability: Consider some $y \notin M$. Then there is $\alpha \in I$ such that $y \notin Z^{\alpha}$. As $\mathcal{S}$ is a chain, it follows that for all $\beta \triangleright \alpha$, we have $y \notin Z^{\beta}$.

For every $\beta \triangleright \alpha$, there is $x^{\beta} \in Z^{\beta}$ such that $x^{\beta} \in f^{\infty}(y)$, since $Z^{\beta}$ satisfies external stability. This defines a net $\left\{x^{\beta}\right\}_{\beta \triangleright \alpha}$. Given that $X$ is compact, it follows by Theorem 2.31 of Aliprantis and Border (2006) that this net has a convergent subnet, say $\left\{x^{\beta^{\prime}}\right\}_{\beta^{\prime} \in I^{\prime}}$, where $I^{\prime} \subseteq I$ is such that for all $\beta \in I$, there is a $\beta^{\prime} \in I^{\prime}$ such that $\beta^{\prime} \triangleright \beta$. Let $\bar{x}$ be the limit of this convergent subnet. We split the remaining part of the proof in two steps. First, we show that $\bar{x} \in M$. Second, we show that $\bar{x} \in f^{\infty}(y)$.

Step 1: $\overline{\boldsymbol{x}} \in \boldsymbol{M}$ : Towards a contradiction, suppose that $\bar{x} \notin M$. Then, there exists $\gamma \in I$ such that $\bar{x} \notin Z^{\gamma}$. In particular, given that $Z^{\gamma}$ is a closed set, there is $\varepsilon>0$ such that $B_{\varepsilon}(\bar{x}) \cap Z^{\gamma}=\emptyset$. Since $\mathcal{S}$ is a chain, we have that $B_{\varepsilon}(\bar{x}) \cap Z^{\delta}=\emptyset$ for all $\delta \triangleright \gamma$. Since $\bar{x}$ is the limit of the subnet $\left\{x^{\beta^{\prime}}\right\}_{\beta^{\prime} \in I^{\prime}}$, there is $\gamma^{\prime} \in I^{\prime}$ such that $\gamma^{\prime} \triangleright \gamma$ and $x^{\gamma^{\prime}} \in B_{\varepsilon}(\bar{x})$. Then we have $x^{\gamma^{\prime}} \in Z^{\gamma^{\prime}}, x^{\gamma^{\prime}} \in B_{\varepsilon}(\bar{x})$, and $B_{\varepsilon}(\bar{x}) \cap Z^{\gamma^{\prime}}=\emptyset$, a contradiction. We conclude that $\bar{x} \in M$.

Step 2: $\overline{\boldsymbol{x}} \in \boldsymbol{f}^{\infty}(\boldsymbol{y})$ : We need to show that for every $\varepsilon>0$ there is $k \in \mathbb{N}$ and $x \in f^{k}(y)$ such that $d(x, \bar{x})<\varepsilon$. 
Let some $\varepsilon>0$ be given. The subnet $\left\{x^{\beta^{\prime}}\right\}_{\beta^{\prime} \in I^{\prime}}$ converges to $\bar{x}$. As such, there exists $\gamma^{\prime} \in I^{\prime}$ such that $d\left(x^{\gamma^{\prime}}, \bar{x}\right)<\varepsilon / 2$. In addition, $x^{\gamma^{\prime}} \in f^{\infty}(y)$, so there is $k \in \mathbb{N}$ and $x \in f^{k}(y)$ such that $d\left(x, x^{\gamma^{\prime}}\right)<\varepsilon / 2$. Then, by the triangle inequality, it holds that

$$
d(x, \bar{x}) \leq d\left(x, x^{\gamma^{\prime}}\right)+d\left(x^{\gamma^{\prime}}, \bar{x}\right)<\varepsilon .
$$

Together with $x \in f^{k}(y)$, this concludes the proof, i.e., $\bar{x} \in f^{\infty}(y)$.

Having established existence of an MSS, we now analyze the cardinality of such sets.

\subsection{Uniqueness}

The first lemma derives a property of the MSS that will be used frequently in the following proofs.

Lemma 4.2. Let $\Gamma$ be a social environment and let $M$ be a myopic stable set of $\Gamma$. For all $x, y \in X$, if $x \in M$ and $y \in f^{\infty}(x)$ then $y \in M$.

Proof. Let $x \in M$ and $y \in f^{\infty}(x)$ and assume, towards a contradiction, that $y \notin M$. Given that $M$ is closed, there is $\varepsilon>0$ such that $B_{\varepsilon}(y) \cap M=\emptyset$. Also, by definition, there is $k \in \mathbb{N}$ and $z \in f^{k}(x)$ such that $z \in B_{\varepsilon}(y)$, i.e. $z \notin M$. Since $z \in f^{k}(x)$, there is a sequence $z^{0}, z^{1}, \ldots, z^{k}$ of length $k$ such that

$$
z^{0}=x, z^{1} \in f\left(z^{0}\right), \ldots, z^{k}=z \in f\left(z^{k-1}\right) .
$$

Let $k^{\prime} \in\{1, \ldots, k\}$ be such that $z^{k^{\prime}}$ is the first element in this sequence with the property that $z^{k^{\prime}} \notin M$. Given that $z^{0}=x \in M$ and $z^{k}=z \notin M$, such an element exists. It holds that $z^{k^{\prime}-1} \in M, z^{k^{\prime}} \in f\left(z^{k^{\prime}-1}\right)$, and $z^{k^{\prime}} \notin M$. This contradicts deterrence of external deviations for $M$.

The following lemma shows that any two myopic stable sets cannot be disjoint.

Lemma 4.3. Let $\Gamma$ be a social environment and let $M_{1}$ and $M_{2}$ be two myopic stable sets of $\Gamma$. Then $M_{1} \cap M_{2} \neq \emptyset$.

Proof. Consider a state $x_{1} \in M_{1}$. If $x_{1} \in M_{2}$, then we are done. Otherwise, by external stability of $M_{2}$ we know that there is $x_{2} \in M_{2}$ such that $x_{2} \in f^{\infty}\left(x_{1}\right)$. Lemma 4.2 tells us that $x_{2} \in M_{1}$, so $x_{2} \in M_{1} \cap M_{2}$.

The following example shows that uniqueness of an MSS cannot be demonstrated without any additional assumptions. 
Example 2. Consider the social environment $\Gamma=\left(\{1\},(X, d), E, \succeq_{1}\right)$, where

$$
X=\{0,1 / 2,1\} \cup\left\{\frac{1}{k} \mid k \in \mathbb{N} \backslash\{1,2\}\right\} \cup\left\{1-\frac{1}{k} \mid k \in \mathbb{N} \backslash\{1,2\}\right\},
$$

and the metric is $d(x, y)=|x-y|$.

The effectivity correspondence is such that the individual can move from both states 0 and 1 to state $1 / 2$ and, for every $k \in \mathbb{N} \backslash\{1,2\}$, from state $1-1 / k$ to state $1 / k$ and from state $1 / k$ to state $1-1 /(k+1)$. The individual cannot make any other moves. The preferences of the individual are such that

$$
\frac{2}{3} \prec_{1} \frac{1}{3} \prec_{1} \frac{3}{4} \prec_{1} \frac{1}{4} \prec_{1} \frac{4}{5} \prec_{1} \frac{1}{5} \prec_{1} \cdots \prec_{1} 1 \prec_{1} 0 \prec_{1} \frac{1}{2}
$$

Now, we claim that both $\{0,1 / 2\}$ and $\{1 / 2,1\}$ are myopic stable sets. It is easy to see that they both satisfy deterrence of external deviations (notice that the individual cannot move from 1 to 0$)$. For external stability, observe that for every $k \in \mathbb{N} \backslash\{1,2\}$ it holds that $0,1 \in f^{\infty}(1 / k)$ and $0,1 \in f^{\infty}(1-1 / k)$. Moreover, it holds that $1 / 2 \in f(0)=f^{\infty}(0)$ and $1 / 2 \in f(1)=f^{\infty}(1)$. Finally, for minimality, the sets $\{0\}$ and $\{1\}$ violate deterrence of external deviations since $1 / 2 \in f(0)$ and $1 / 2 \in f(1)$. The set $\{1 / 2\}$ violates external stability as $1 / 2 \notin f^{\infty}(x)$ for any $x \in X$ different from $0,1 / 2$ and 1 .

Although Example 2 shows that the MSS is not necessarily unique, we can restore uniqueness by imposing the following mild continuity assumption on the dominance correspondence $f$.

Definition 4.4 (Lower Hemi-continuity of $f$ ). The dominance correspondence $f: X \rightarrow X$ is lower hemi-continuous if for every sequence $\left\{x^{k}\right\}_{k \in \mathbb{N}}$ in $X$ such that $x^{k} \rightarrow x$ and for every $y \in f(x)$ there is a sequence $\left\{y^{k}\right\}_{k \in \mathbb{N}}$ in $X$ such that for all $k, y^{k} \in f\left(x^{k}\right)$ and $y^{k} \rightarrow y$.

In words, if there is a sequence of states converging to $x$ and $y$ dominates $x$, then it is possible to find a sequence of states that converges to $y$ such that each element in this sequence dominates the corresponding element of the sequence that converges to $x$. Later on, we will show that this condition is always satisfied if preferences are continuous and some continuity condition on the effectivity relation is satisfied. The following technical lemma is helpful in proving uniqueness of an MSS.

Lemma 4.5. If the dominance correspondence $f: X \rightarrow X$ is lower hemi-continuous, then the asymptotic dominance correspondence $f^{\infty}: X \rightarrow X$ is transitive.

Proof. Let $x, y, z \in X$ be such that $y \in f^{\infty}(x)$ and $z \in f^{\infty}(y)$. We have to show that $z \in f^{\infty}(x)$, so we need to show that for every $\varepsilon>0$, there is $k^{\prime} \in \mathbb{N}$ and $z^{\prime} \in f^{k^{\prime}}(x)$ such that $d\left(z^{\prime}, z\right)<\varepsilon$. 
By assumption, $z \in f^{\infty}(y)$, so there is $k \in \mathbb{N}$ and $z_{1} \in f^{k}(y)$ such that $d\left(z_{1}, z\right)<\varepsilon / 2$. In addition, as $y \in f^{\infty}(x)$, we know that for every $\ell \in \mathbb{N}$ there is $k_{\ell} \in \mathbb{N}$ and $y^{\ell} \in f^{k_{\ell}}(x)$ such that $d\left(y^{\ell}, y\right)<1 / \ell$. This generates a sequence $\left\{y^{\ell}\right\}_{\ell \in \mathbb{N}}$ that converges to $y$, i.e., $y^{\ell} \rightarrow y$.

Note that $f^{k}$ is lower hemi-continuous, since it is a composition of $k$ lower hemi-continuous correspondences. Given lower hemi-continuity of $f^{k}$ and the fact that $z_{1} \in f^{k}(y)$, we know that there is a sequence $\left\{z_{2}^{\ell}\right\}_{\ell \in \mathbb{N}}$ such that $z_{2}^{\ell} \rightarrow z_{1}$ and $z_{2}^{\ell} \in f^{k}\left(y^{\ell}\right)$. Now, we have that $y^{\ell} \in f^{k_{\ell}}(x)$ and $z_{2}^{\ell} \in f^{k}\left(y^{\ell}\right)$, which gives $z_{2}^{\ell} \in f^{k+k_{\ell}}(x)$.

Take $\ell$ large enough such that $d\left(z_{2}^{\ell}, z_{1}\right)<\varepsilon / 2$. Conclude that $z_{2}^{\ell} \in f^{k+k_{\ell}}(x)$ and

$$
d\left(z_{2}^{\ell}, z\right) \leq d\left(z_{2}^{\ell}, z_{1}\right)+d\left(z_{1}, z\right)<\varepsilon
$$

This completes the proof.

We are now ready to establish the uniqueness of an MSS whenever the dominance correspondence $f$ is lower hemi-continuous.

Theorem 4.6. Let $\Gamma$ be a social environment such that the corresponding dominance correspondence $f$ is lower hemi-continuous. Then $\Gamma$ has a unique MSS.

Proof. Suppose not, then, by Theorem 4.1 and Lemma 4.3, there exists an MSS $M_{1}$ and an MSS $M_{2}$ such that $M_{1} \neq M_{2}$ and their intersection $M_{3}=M_{1} \cap M_{2}$ is non-empty. Let us show that $M_{3}$ is a QMSS, contradicting the minimality of $M_{1}$ and $M_{2}$, and establishing the uniqueness of the MSS. First of all, notice that $M_{3}$, being the intersection of two closed sets, is also closed.

For deterrence of external deviations, let $x \in M_{3}$ and, towards a contradiction, suppose that $y \in f(x)$ and $y \notin M_{3}$. Then given that $x \in M_{1}$ and $M_{1}$ satisfies deterrence of external deviations, it must be that $y \in M_{1}$. Also given that $x \in M_{2}$ and $M_{2}$ satisfies deterrence of external deviations, it must be that $y \in M_{2}$. This implies that $y \in M_{1} \cap M_{2}=M_{3}$, a contradiction. Consequently, $M_{3}$ satisfies deterrence of external deviations.

For external stability, take any $y \notin M_{3}$. There are three cases to consider.

Case 1: $\boldsymbol{y} \in \boldsymbol{M}_{\mathbf{1}} \backslash \boldsymbol{M}_{\mathbf{3}}$ : Then, by external stability of $M_{2}$, there is $x \in M_{2}$ such that $x \in f^{\infty}(y)$. By Lemma 4.2, we have that $x \in M_{1}$. This means that $x \in M_{2} \cap M_{1}=M_{3}$ what we needed to show.

Case 2: $\boldsymbol{y} \in \boldsymbol{M}_{\mathbf{2}} \backslash \boldsymbol{M}_{\mathbf{3}}$ : The proof is symmetric to Case 1 with $M_{1}$ and $M_{2}$ interchanged.

Case 3: $\boldsymbol{y} \in \boldsymbol{X} \backslash\left(\boldsymbol{M}_{\mathbf{1}} \cup \boldsymbol{M}_{\mathbf{2}}\right)$ : We know, by external stability of $M_{1}$, that there is $x \in M_{1}$ such that $x \in f^{\infty}(y)$. If $x \in M_{3}$, we are done. If not, we know from Case 1 above that there is $z \in M_{3}$ such that $z \in f^{\infty}(x)$. It follows from $x \in f^{\infty}(y)$ and $z \in f^{\infty}(x)$ that $z \in f^{\infty}(y)$ by Lemma 4.5 . 
The continuity condition of Theorem 4.6 is trivially satisfied when the state space $X$ is finite. As such, for all applications with a finite state space, we have uniqueness of the MSS.

The dominance correspondence $f$ is defined in terms of the individual preference relations $\left(\succeq_{i}\right)_{i \in N}$ and the effectivity correspondence $E$. It might therefore be difficult to verify lower hemi-continuity of $f$ directly. We therefore provide conditions on the primitives of a social environment that imply lower hemi-continuity of $f$. As a first condition, we impose continuity of the preferences.

Definition 4.7 (Continuity of Preferences). The preference relation $\succeq_{i}$ of individual $i \in N$ is continuous if for any two sequences $\left\{x^{k}\right\}_{k \in \mathbb{N}}$ and $\left\{y^{k}\right\}_{k \in \mathbb{N}}$ in $X$ with $x^{k} \rightarrow x$ and $y^{k} \rightarrow y$ and, for every $k \in \mathbb{N}, x^{k} \succeq_{i} y^{k}$, it holds that $x \succeq_{i} y$.

Our second condition is lower hemi-continuity of the effectivity correspondence $E$. Towards this end, consider, for every $S \in \mathcal{N}$, the correspondence $G_{S}: X \rightarrow X$ defined by

$$
G_{S}(x)=\{x\} \cup\{y \in X \mid S \in E(x, y)\}, \quad x \in X,
$$

which associates to every state $x \in X$ the set of states coalition $S$ can move to together with state $x$ itself.

Definition 4.8 (Lower Hemi-continuity of $E$ ). The effectivity correspondence $E$ is lower hemi-continuous if for every coalition $S \in \mathcal{N}$ the correspondence $G_{S}: X \rightarrow X$ is lower hemicontinuous, i.e., for every sequence $\left\{x^{k}\right\}_{k \in \mathbb{N}}$ in $X$ such that $x^{k} \rightarrow x$ and for every $y \in G_{S}(x)$ there is a sequence $\left\{y^{k}\right\}_{k \in \mathbb{N}}$ such that $y^{k} \in G_{S}\left(x^{k}\right)$ and $y^{k} \rightarrow y$.

Theorem 4.9 shows that continuity of preferences and lower hemi-continuity of $E$ is sufficient for the dominance correspondence $f$ to be lower hemi-continuous.

Theorem 4.9. Let $\Gamma$ be a social environment such that the preferences $\left(\succeq_{i}\right)_{i \in N}$ are continuous and the effectivity correspondence $E$ is lower hemi-continuous. Then the dominance correspondence $f$ is lower hemi-continuous.

Proof. Let $x, y \in X$ and sequences $\left\{x^{k}\right\}_{k \in \mathbb{N}}$ and $\left\{y^{k}\right\}_{k \in \mathbb{N}}$ in $X$ be given. Let us first show that if individual $i \in N$ strictly prefers $y$ to $x, y \succ_{i} x$, then there is a number $\ell \in \mathbb{N}$ such that for all $k \geq \ell, y^{k} \succ_{i} x^{k}$. Suppose not, then for every $\ell \in \mathbb{N}$ we can find $k_{\ell} \geq \ell$ such that $x^{k_{\ell}} \succeq_{i} y^{k_{\ell}}$. This creates sequences $\left\{x^{k_{\ell}}\right\}_{\ell \in \mathbb{N}},\left\{y^{k_{\ell}}\right\}_{\ell \in \mathbb{N}}$ in $X$ with $x^{k_{\ell}} \rightarrow x$ and $y^{k_{\ell}} \rightarrow y$ such that $x^{k_{\ell}} \succeq_{i} y^{k_{\ell}}$. By continuity of $\succeq_{i}, x \succeq_{i} y$, a contradiction.

Let $\left\{x^{k}\right\}_{k \in \mathbb{N}}$ be a sequence in $X$ such that $x^{k} \rightarrow x \in X$ and consider some $y \in f(x)$. Then either $y=x$ or $y \neq x$ and there is a coalition $S$ such that $S \in E(x, y)$ and $y \succ_{i} x$ for all $i \in S$.

If $y=x$, take the sequence $\left\{y^{k}\right\}_{k \in \mathbb{N}}$ in $X$ defined by $y^{k}=x^{k}$. We immediately have that, for every $k \in \mathbb{N}, y^{k} \in f\left(x^{k}\right)$ and $y^{k} \rightarrow y$. 
If $y \neq x$ and there is a coalition $S$ such that $S \in E(x, y)$ and $y \succ_{i} x$ for all $i \in S$, we need to show that there is a sequence $\left\{y^{k}\right\}_{k \in \mathbb{N}}$ such that for all $k, y^{k} \in f\left(x^{k}\right)$ and $y^{k} \rightarrow y$. By lower hemi-continuity of the correspondence $G_{S}$, we know that there is a sequence $\left\{y^{k}\right\}_{k \in \mathbb{N}}$ such that $y^{k} \in G_{S}\left(x^{k}\right)$ and $y^{k} \rightarrow y$. By the first paragraph of the proof, we know that for every $i \in N$ there is $\ell_{i} \in \mathbb{N}$ such that $y^{k} \succ_{i} x^{k}$ for all $k \geq \ell_{i}$. Let $\ell=\max _{i \in S} \ell_{i}$. Then, for every $k \geq \ell$ and every $i \in S, y^{k} \succ_{i} x^{k}$ and $S \in E\left(x^{k}, y^{k}\right)$, which shows that $y^{k} \in f\left(x^{k}\right)$. The sequence $\left\{z^{k}\right\}_{k \in \mathbb{N}}$ defined by $z^{k}=x^{k}$ if $k<\ell$ and $z^{k}=y^{k}$ if $k \geq \ell$ therefore has all the desired properties.

Combining Theorem 4.6 and Lemma 4.9 directly yields the following corollary which gives a sufficient condition on the primitives of the model to obtain a unique MSS.

Corollary 4.10. Let $\Gamma$ be a social environment such that the preferences $\left(\succeq_{i}\right)_{i \in N}$ are continuous and the effectivity correspondence $E$ is lower hemi-continuous. Then there is a unique MSS.

\subsection{Closed Cycles and the Core}

We finish this section with two general results about the structure of an MSS.

Definition 4.11 (Closed Cycle). A closed cycle of a social environment $\Gamma$ is a set $C \subseteq X$ such that for every $x \in C$ it holds that $f^{\infty}(x)=C$.

Intuitively, a closed cycle is a subset of $X$ which is closed under the asymptotic dominance correspondence $f^{\infty}$. We denote the union of all closed cycles by CC, so CC contains all the states that are part of some closed cycle. The following result characterizes the MSS for finite environments as the union of all closed cycles and shows that this union is a subset of the MSS for social environments with an infinite state space.

Theorem 4.12. Let $\Gamma$ be a social environment and $M$ be an MSS of $\Gamma$. It holds that $\mathrm{CC} \subseteq M$. If $X$ is finite, we have $\mathrm{CC}=M$.

Proof. Towards a contradiction, suppose there is a closed cycle $C$ which is not a subset of $M$. Let $x \in C$ and $x \notin M$. By external stability there is $y \in M$ such that $y \in f^{\infty}(x)$. As $x \in C$, we also have that $x \in f^{\infty}(y)$. By Lemma 4.2, it follows that $x \in M$, a contradiction. Since the choice of $C$ was arbitrary, we have shown that $\mathrm{CC} \subseteq M$.

We show next that if $X$ is finite, then $\mathrm{CC}=M$. Since $\mathrm{CC} \subseteq M$, we only need to show that $\mathrm{CC}$ is a QMSS. The set CC satisfies deterrence of external deviations, since for all $x \in \mathrm{CC}, f(x) \subseteq f^{\infty}(x) \subseteq \mathrm{CC}$. It remains to verify external stability of CC, i.e., for every state $x \notin \mathrm{CC}, f^{\infty}(x) \cap \mathrm{CC} \neq \emptyset$. 
Let $x \notin \mathrm{CC}$ and define $Y=f^{\infty}(x)$. Note that $Y$ is non-empty since $x \in f(x)$, finite and that $f^{\infty}(y) \subseteq Y$ for every $y \in Y$. Let us represent the set $Y$ and the dominance relation $f$ on $Y$ by a finite directed graph $D$, i.e., (i) $Y$ are the vertices of $D$ and (ii) $D$ has an arc from $y$ to $z$ if and only if $z \in f(y)$. By contracting each strongly connected component of $D$ to a single vertex, we obtain a directed acyclic graph, which is called the condensation of $D$. As the condensation is finite and acyclic, it has a maximal element, say $c$. Observe that $c$ represents a closed cycle $C$, so $Y \cap \mathrm{CC} \neq \emptyset$.

A sink is a closed cycle which consists of only one state, i.e., $f(x)=x$. The union of all sinks is called the core.

Definition 4.13 (Core). Let $\Gamma$ be a social environment. The core of $\Gamma$ is given by

$$
\mathrm{CO}=\{x \in X \mid f(x)=\{x\}\}
$$

It is well-known that the core may be empty for some social environments. However, if it is not empty, then it is always contained in the myopic stable set by virtue of Theorem 4.12 .

Corollary 4.14. Let $\Gamma$ be a social environment and let $M$ be an MSS. Then we have $\mathrm{CO} \subseteq M$.

The next definition is inspired by the finite analogue for normal-form games as presented in Friedman and Mezzetti (2001).

Definition 4.15 (Weak (Finite) Improvement Property). A social environment $\Gamma$ satisfies the weak finite improvement property if for each state $x \in X, f^{\mathbb{N}}(x)$ contains a sink and the weak improvement property if for each state $x \in X, f^{\infty}(x)$ contains a sink.

The following provides a characterization for the MSS in social environments with the weak improvement property.

Theorem 4.16. Let $\Gamma$ be a social environment and let $f$ be lower hemi-continuous. Then, the MSS of $\Gamma$ is equal to the core if and only if the social environment satisfies the weak improvement property.

Proof. Assume that $\Gamma$ has the weak improvement property. By Corollary 4.14, CO $\subseteq M$. We will show that $\mathrm{CO}$ is a QMSS. By minimality, it then follows that $\mathrm{CO}=M$.

In order to see that CO is closed let $\left\{x^{k}\right\}_{k \in \mathbb{N}}$ be a sequence in CO, i.e., for all $k,\left\{x^{k}\right\}=$ $f\left(x^{k}\right)$. Now assume that $x^{k} \rightarrow x$ and $x \notin$ CO. This means that there is $y \neq x$ such that $y \in f(x)$. By lower hemi-continuity of $f$, there should be a sequence $\left\{y^{k}\right\}_{k \in \mathbb{N}}$ such $y^{k} \in f\left(x^{k}\right)$ and $y^{k} \rightarrow y$. As for all $k, x^{k} \in \mathrm{CO}$, we have that for all $k, y^{k}=x^{k}$ which means that $y^{k} \rightarrow x \neq y$, a contradiction. Deterrence of external deviations is immediate for the core 
as it is the union of sinks. If the social environment satisfies the weak improvement property we have that for all $x \notin \mathrm{CO}, f^{\infty}(x) \cap \mathrm{CO} \neq \emptyset$ thus the core satisfies external stability.

For the reverse, assume that $\mathrm{CO}=M$. Now, if $x \in M$, it is a sink, so $f^{\infty}(x)=\{x\} \subseteq \mathrm{CO}$. If $x \notin \mathrm{CO}$ we have by external stability of $M$, that $f^{\infty}(x) \cap M \neq \emptyset$, so $f^{\infty}(x)$ contains a sink. This shows that $\Gamma$ satisfies the weak improvement property.

The requirement of lower hemi-continuity of $f$ in Theorem 4.16 can be weakened to the requirement that $\mathrm{CO}$ should be closed.

\section{Applications}

In this section, we discuss how the results from the previous section can be applied to the examples in Section 2.

\subsection{Coalition Function Form Games}

We can associate a social environment $\Gamma=\left(N,(X, d), E,\left(\succeq_{i}\right)_{i \in N}\right)$ to each coalition function form game $(N, v)$ as in Section 2.1, so we impose the properties of non-interference, full support and the $\gamma$-model.

By Theorem 4.1 we know that there exists at least one non-empty MSS. Let us first show that for coalition function form games, the MSS is also unique. Towards this end, we first show that the preference relations $\succeq_{i}$ are continuous and that the effectivity correspondence $E$ is lower hemi-continuous.

Lemma 5.1. Let $(N, v)$ be a coalition function form game and let $\Gamma=\left(N,(X, d), E,\left(\succeq_{i}\right)_{i \in N}\right)$ be the induced social environment as in Section 2.1. Then, for every $i \in N$, the preference relation $\succeq_{i}$ is continuous and the effectivity correspondence $E$ is lower hemi-continuous.

Proof. Let some $i \in N$ be given. To show continuity of $\succeq_{i}$, let $\left\{x^{k}\right\}_{k \in \mathbb{N}}$ and $\left\{y^{k}\right\}_{k \in \mathbb{N}}$ be sequences in $X$ such that $x^{k} \rightarrow x$ and $y^{k} \rightarrow y$. Then, by the continuity of $u_{i}$, we have that $u_{i}\left(x^{k}\right) \rightarrow u_{i}(x)$ and $u_{i}\left(y^{k}\right) \rightarrow u_{i}(y)$. So if $u_{i}\left(x^{k}\right) \geq u_{i}\left(y^{k}\right)$ for all $k \in \mathbb{N}$, we obtain $u_{i}(x) \geq u_{i}(y)$, which shows that $x \succeq_{i} y$.

To show lower hemi-continuity of $E$, let some $S \in \mathcal{N}$, a sequence $\left\{x^{k}\right\}_{k \in \mathbb{N}}$ in $X$ such that $x^{k} \rightarrow x$ and some $y \in G_{S}(x)$ be given. We show that there is a sequence $\left\{y^{k}\right\}_{k \in \mathbb{N}}$ such that $y^{k} \in G_{S}\left(x^{k}\right)$ and $y^{k} \rightarrow y$. If $y=x$, then the choice $y^{k}=x^{k}$ would do, so consider the case $y \neq x$

First of all, there is $k^{\prime} \in \mathbb{N}$ such that for all $k \geq k^{\prime}, \pi\left(x^{k}\right)=\pi(x)$, so in particular $\mathcal{U}\left(x^{k}, S\right)=\mathcal{U}(x, S)$. For every $k<k^{\prime}$, we define $y^{k}=x^{k}$. For every $k \geq k^{\prime}$, we define $y^{k} \in X$ 
by $\pi\left(y^{k}\right)=\pi(y)$ and

$$
u_{i}\left(y^{k}\right)= \begin{cases}u_{i}(y), & i \in N \backslash U(x, S), \\ u_{i}\left(x^{k}\right), & i \in U(x, S) .\end{cases}
$$

Consider some $k \geq k^{\prime}$. Since $y \neq x$, it holds that $S \in \pi(y)$ and, for every $i \in N \backslash(S \cup U(x, S))$, we have that $i$ is a residual player and the properties of the $\gamma$-model imply that $\{i\} \in \pi(y)$. The same properties hold for $\pi\left(y^{k}\right)$. For every $i \in S$, it holds that $u_{i}\left(y^{k}\right)=u_{i}(y)$, so $u_{i}\left(y^{k}\right) \geq v(\{i\})$ and $\sum_{i \in S} u_{i}\left(y^{k}\right)=v(S)$. For every $i \in N \backslash(S \cup U(x, S))$, we have that $u_{i}\left(y^{k}\right)=v(\{i\})=u_{i}(y)$. For every $i \in U(x, S)$ it holds that $u_{i}(y)=u_{i}(x)$ and $u_{i}\left(y^{k}\right)=u_{i}\left(x^{k}\right)$. By coalitional sovereignty, we have that $y^{k} \in G_{S}\left(x^{k}\right)$. Using that $x^{k} \rightarrow x$, it follows easily that $y^{k} \rightarrow y$.

Lemma 5.1 together with Theorem 4.6 and Lemma 4.9 shows uniqueness of the MSS.

Corollary 5.2. Let $(N, v)$ be a coalition function form game and let $\Gamma$ be the induced social environment as in Section 2.1. Then $\Gamma$ has a unique MSS.

In fact, most other models of coalitional sovereignty will also lead to lower hemi-continuity of $E$ so will also have a unique MSS. However, establishing the lower hemi-continuity of $E$ must be done case by case.

The Coalition Structure Core One of the most prominent set-valued solution concepts for coalition function form games is the coalition structure core.

Definition 5.3 (Coalition Structure Core). Let $(N, v)$ be a coalition function form game and let $\Gamma=\left(N,(X, d), E,\left(\succeq_{i}\right)_{i \in N}\right)$ be the induced social environment as in Section 2.1. The coalition structure core of $(N, v)$ is the set of states $x \in X$ such that for every coalition $S \in \mathcal{N}$

$$
\sum_{i \in S} u_{i}(x) \geq v(S)
$$

In words, the coalition structure core gives to the members of each coalition at least the payoff they can obtain by forming that coalition.

Lemma 5.4. Let $(N, v)$ be a coalition function form game and let $\Gamma=\left(N,(X, d), E,\left(\succeq_{i}\right)_{i \in N}\right)$ be the induced social environment as in Section 2.1. The coalition structure core of $(N, v)$ is equal to the core of $\Gamma$.

Proof. Let $Y$ be the coalition structure core. Let $y \in \mathrm{CO}$ and assume $y \notin Y$. Then there is a coalition $S$ such that $\sum_{i \in S} u_{i}(y)<v(S)$. Since $y \in X$, it holds for all $i \in S, u_{i}(y) \geq v(\{i\})$. 
Now, let $u_{S}$ be a vector of payoffs for the members in $S$ such that $\sum_{i \in S} u_{i}=v(S)$ and for all $i \in S, u_{i}>u_{i}(y)$. Then, by full support, there exists a state $y^{\prime} \in X$ such that $S \in E\left(y, y^{\prime}\right)$ and $u_{S}=u_{S}\left(y^{\prime}\right)$. Conclude that $y^{\prime} \in f(y)$. This contradicts the fact that $y \in \mathrm{CO}$.

For the reverse, let $y \in Y$ and $z \in f(y)$ such that $z \neq y$, i.e., $y \notin \mathrm{CO}$. Then there is $S \in E(y, z)$ such that $u_{S}(z) \gg u_{S}(y)$. Also,

$$
v(S)=\sum_{i \in S} u_{i}(z)>\sum_{i \in S} u_{i}(y) \geq v(S)
$$

where the first equality follows from the definition of the state space and the last inequality from the definition of $Y$. We have obtained a contradiction.

Kóczy and Lauwers (2004) define the coalition structure core to be accessible if from any initial state there is a finite sequence of states ending with an element of the coalition structure core and each element in that sequence outsider independently dominates the previous element. The notion of outsider independent domination differs from our notion of a myopic improvement in two ways. First, residual players are not required to become singletons after a move has taken place. Second, improvements for the members of the coalition that moves are not necessarily strict improvements.

The following example illustrates that under the requirement of strict improvements of all members involved in a move, as in our dominance correspondence $f$, the coalition structure core does not satisfy strong external stability, i.e., it is not the case that for all states $x \in X$, there is a state $y$ in the coalitional structure core such that $y \in f^{\mathbb{N}}(x)$.

Example 3. Let $(N, v)$ be a coalition function form game such that $N=\{1,2,3\}, v(\{1,2\})=$ 1 , and $v(\{2,3\})=1$. All other coalitions have a worth of 0 . Here, player 2 can choose to form a coalition with either player 1 or player 3 to form a two-person coalition generating a surplus equal to one. The coalition structure core therefore consists of only two states, $y$ and $y^{\prime}$, with equal payoffs, $u(y)=u\left(y^{\prime}\right)=(0,1,0)$, and coalitional structures $\pi(y)=\{\{1,2\},\{3\}\}$, and $\pi\left(y^{\prime}\right)=\{\{1\},\{2,3\}\}$.

Consider an initial state $x^{0} \in X$ such that $\pi\left(x^{0}\right)=\{\{1\},\{2\},\{3\}\}$ and $u\left(x^{0}\right)=(0,0,0)$. Under our notion of a myopic improvement, where all players involved in a move have to gain strictly, a state $x^{1}$ belongs to $f\left(x^{0}\right)$ if and only if either $\pi\left(x^{1}\right)=\{\{1,2\},\{3\}\}$ and $u\left(x^{1}\right)=(\varepsilon, 1-\varepsilon, 0)$ for some $\varepsilon \in(0,1)$ or $\pi\left(x^{1}\right)=\{\{1\},\{2,3\}\}$ and $u\left(x^{1}\right)=(0,1-\varepsilon, \varepsilon)$ for some $\varepsilon \in(0,1)$. It follows that $x^{1}$ is a state where either player 1 or player 3 receives a payoff of zero and the other two players receive a strictly positive payoff summing up to 1 .

Now consider any state $x^{k}$ such that either player 1 or player 3 receives 0 and the other two players receive a strictly positive payoff summing up to 1 . We claim that any state $x^{k+1} \in f\left(x^{k}\right)$ has the same properties. Without loss of generality, assume that $u_{3}\left(x^{k}\right)=0$. Let $x^{k+1}$ be an element of $f\left(x^{k}\right)$ different from $x^{k}$. Since $u_{1}\left(x^{k}\right)+u_{2}\left(x^{k}\right)=1$, the only 
coalition that can move is $\{2,3\}$ and it holds that $\pi\left(x^{k+1}\right)=\{\{1\},\{2,3\}\}$. Moreover, it must also hold that $u_{2}\left(x^{k+1}\right)>u_{2}\left(x^{k}\right)>0$ and $u_{3}\left(x^{k+1}\right)>u_{3}\left(x^{k}\right)=0$, which proves the claim. It now follows that for every $k \in \mathbb{N}$, if $x^{k} \in f^{k}\left(x^{0}\right)$, then $x^{k}$ is such that there are two players with a strictly positive payoff. Given this, there is no $k \in \mathbb{N}$ such that $x^{k}$ belongs to the coalition structure core.

Theorem 5.5 shows that the MSS coincides with the coalition structure core whenever it is non-empty.

Theorem 5.5. Let $(N, v)$ be a coalition function form game, $\Gamma$ be the induced social environment as in Section 2.1, and $Y$ be the coalition structure core of $\Gamma$. If $Y$ is non-empty, then the unique MSS of $\Gamma$ is equal to $Y$.

Proof. From Lemma 5.1 we know that $f$ is lower hemi-continuous. Also Lemma 5.4 shows that $Y$ is equal to the core of $\Gamma$. If we can show that $\Gamma$ satisfies the weak improvement property whenever $Y \neq \emptyset$, then we can use Theorem 4.16 to establish our proof. Since the proof is trivial when the number of individuals $n=1$, we assume $n \geq 2$ throughout.

So assume that $Y \neq \emptyset$. We need to show that for all $x^{0} \in X, f^{\infty}\left(x^{0}\right) \cap Y \neq \emptyset$. If $x^{0}$ in $Y$, then nothing needs to be shown, so assume that $x^{0} \in X \backslash Y$. We need to show that for every $\varepsilon>0$ there is a number $k^{\prime} \in \mathbb{N}$, a state $x^{k^{\prime}} \in f^{k^{\prime}}\left(x^{0}\right)$, and a state $y \in Y$ such that $d\left(x^{k^{\prime}}, y\right)<\varepsilon$.

Let some $\varepsilon>0$ be given. Béal, Rémila, and Solal (2013) show that there exists a sequence of states $\left(x^{0}, \ldots, x^{k^{\prime}}\right)$ such that $x^{k^{\prime}} \in Y, k^{\prime}$ is less than or equal to $\left(n^{2}+4 n\right) / 4$, and, for every $k \in\left\{1, \ldots, k^{\prime}\right\}$,

1. there is $S^{k} \in \mathcal{N}$ such that $S^{k} \in E\left(x^{k-1}, x^{k}\right)$,

2. $u_{S^{k}}\left(x^{k-1}\right)<u_{S^{k}}\left(x^{k}\right)$.

Notice that the inequality in 2. only means that at least one of the players in $S^{k}$ gets a strictly higher payoff, though not necessarily all of them. Let $P^{k}$ be the set of partners of the players in $S^{k}$ at state $x^{k-1}$, more formally defined as

$$
P^{k}=\cup_{\left\{S \in \pi\left(x^{k-1}\right) \mid S \cap S^{k} \neq \emptyset\right\}} S,
$$

so $P^{k}$ is equal to the moving coalition $S^{k}$ together with the residual players. Since $S^{k} \in$ $E\left(x^{k-1}, x^{k}\right)$, it follows that

$$
\begin{array}{ll}
u_{i}\left(x^{k}\right)=v(\{i\}), & i \in P^{k} \backslash S^{k}, \\
u_{i}\left(x^{k}\right)=u_{i}\left(x^{k-1}\right), & i \in N \backslash P^{k} .
\end{array}
$$


We define $W^{k} \subset S^{k}$ to be the, possibly empty, proper subset of $S^{k}$ consisting of players that only weakly improve when moving from state $x^{k-1}$ to state $x^{k}$, so for every $i \in W^{k}$ it holds that $u_{i}\left(x^{k-1}\right)=u_{i}\left(x^{k}\right)$. We define

$$
\begin{aligned}
\delta & =\min _{k \in\left\{1, \ldots, k^{\prime}\right\}} \min _{i \in S^{k} \backslash W^{k}} u_{i}\left(x^{k}\right)-u_{i}\left(x^{k-1}\right), \\
\varepsilon^{\prime} & =\min \{\delta, \varepsilon\},
\end{aligned}
$$

so $\delta$ is the smallest improvement of any of the strictly improving players involved in any move along the sequence. It holds that $\delta>0$ and therefore that $\varepsilon^{\prime}>0$. For $k \in\left\{0, \ldots, k^{\prime}\right\}$, define

$$
\nu_{k}=\frac{n^{2 k}}{n^{2 k^{\prime}+1}} .
$$

We define $e\left(W^{k}\right)=0$ if $W^{k}=\emptyset$ and $e\left(W^{k}\right)=1$ otherwise. We use the sequence $\left(x^{0}, x^{1}, \ldots, x^{k^{\prime}}\right)$ of states as constructed by Béal, Rémila, and Solal (2013) to define a new sequence $\left(\widetilde{x}^{0}, \widetilde{x}^{1}, \ldots, \widetilde{x}^{k^{\prime}}\right)$ of states by setting $\widetilde{x}^{0}=x^{0}$ and, for every $k \in\left\{1, \ldots, k^{\prime}\right\}$,

$$
\begin{aligned}
& \pi\left(\widetilde{x}^{k}\right)=\pi\left(x^{k}\right), \\
& u_{i}\left(\widetilde{x}^{k}\right)=u_{i}\left(x^{k}\right)+\varepsilon^{\prime} \nu_{k} \frac{\left|S^{k} \backslash W^{k}\right|}{\left|W^{k}\right|}, \quad i \in W^{k}, \\
& u_{i}\left(\widetilde{x}^{k}\right)=u_{i}\left(x^{k}\right)-\varepsilon^{\prime} \nu_{k} e\left(W^{k}\right), \quad i \in S^{k} \backslash W^{k}, \\
& u_{i}\left(\widetilde{x}^{k}\right)=u_{i}\left(x^{k}\right)=v(\{i\}), \quad i \in P^{k} \backslash S^{k} \text {, } \\
& u_{i}\left(\widetilde{x}^{k}\right)=u_{i}\left(\widetilde{x}^{k-1}\right), \quad i \in N \backslash P^{k} \text {. }
\end{aligned}
$$

Notice that the first line does not entail a division by zero, since if $i \in W^{k}$, then $W^{k} \neq \emptyset$.

Compared to the sequence $\left(x^{0}, x^{1}, \ldots, x^{k^{\prime}}\right)$, the sequence $\left(\widetilde{x}^{0}, \widetilde{x}^{1}, \ldots, \widetilde{x}^{k^{\prime}}\right)$ is such that each strictly improving player in $S^{k} \backslash W^{k}$ donates an amount $\varepsilon^{\prime} \nu_{k} /\left|W^{k}\right|$ to each of the players in $W^{k}$ whenever the latter set is non-empty. It is also important to observe that the fraction $\nu_{k}$ is an $n^{2}$ multiple of $\nu_{k-1}$ and that $\nu_{k^{\prime}}=1 / n$.

We show first by induction that, for every $k \in\left\{0, \ldots, k^{\prime}\right\}, \widetilde{x}^{k} \in X$. Obviously, it holds that $\widetilde{x}^{0}=x^{0} \in X$. Assume that, for some $k \in\left\{1, \ldots, k^{\prime}\right\}, \widetilde{x}^{k-1} \in X$. We show that $\widetilde{x}^{k} \in X$. It holds that

$$
\begin{array}{ll}
u_{i}\left(\widetilde{x}^{k}\right)>u_{i}\left(x^{k}\right) \geq v(\{i\}), & i \in W^{k}, \\
u_{i}\left(\widetilde{x}^{k}\right) \geq u_{i}\left(x^{k-1}\right)+\delta-\varepsilon^{\prime} \nu_{k}>u_{i}\left(x^{k-1}\right)+\delta-\varepsilon^{\prime} \geq u_{i}\left(x^{k-1}\right) \geq v(\{i\}), & i \in S^{k} \backslash W^{k}, \\
u_{i}\left(\widetilde{x}^{k}\right)=v(\{i\}), & i \in P^{k} \backslash S^{k}, \\
u_{i}\left(\widetilde{x}^{k}\right)=u_{i}\left(\widetilde{x}^{k-1}\right) \geq v(\{i\}), & i \in N \backslash P^{k},
\end{array}
$$

where the very last inequality follows from the induction hypothesis. Moreover, for every $S \in \pi\left(x^{k}\right)$, it holds that either $S=S^{k}$ and $W^{k}=\emptyset$, so

$$
\sum_{i \in S} u_{i}\left(\widetilde{x}^{k}\right)=\sum_{i \in S^{k}} u_{i}\left(x^{k}\right)=v(S)
$$


or $S=S^{k}$ and $W^{k} \neq \emptyset$, so

$$
\sum_{i \in S} u_{i}\left(\widetilde{x}^{k}\right)=\sum_{i \in W^{k}}\left(u_{i}\left(x^{k}\right)+\varepsilon^{\prime} \nu_{k} \frac{\left|S^{k} \backslash W^{k}\right|}{\left|W^{k}\right|}\right)+\sum_{i \in S^{k} \backslash W^{k}}\left(u_{i}\left(x^{k}\right)-\varepsilon^{\prime} \nu_{k}\right)=\sum_{i \in S^{k}} u_{i}\left(x^{k}\right)=v(S),
$$

or $S=\left\{i^{\prime}\right\}$ with $i^{\prime} \in P^{k} \backslash S^{k}$ and

$$
\sum_{i \in S} u_{i}\left(\widetilde{x}^{k}\right)=u_{i^{\prime}}\left(\widetilde{x}^{k}\right)=u_{i^{\prime}}\left(x^{k}\right)=v\left(\left\{i^{\prime}\right\}\right)=v(S)
$$

or $S \subseteq N \backslash P^{k}$, so $S \in \pi\left(\widetilde{x}^{k-1}\right)$, and

$$
\sum_{i \in S} u_{i}\left(\widetilde{x}^{k}\right)=\sum_{i \in S} u_{i}\left(\widetilde{x}^{k-1}\right)=v(S)
$$

where the last equality makes use of the induction hypothesis. We have now completed the proof of the fact that for every $k \in\left\{0, \ldots, k^{\prime}\right\}, \widetilde{x}^{k} \in X$.

We show next by induction that, for every $k \in\left\{0, \ldots, k^{\prime}\right\}$, and for every $i \in N$,

$$
\left|u_{i}\left(\widetilde{x}^{k}\right)-u_{i}\left(x^{k}\right)\right| \leq \varepsilon^{\prime} \nu_{k}(n-1) .
$$

Obviously, for every $i \in N$, it holds that $\left|u_{i}\left(\widetilde{x}^{0}\right)-u_{i}\left(x^{0}\right)\right|=0 \leq \varepsilon^{\prime} \nu_{0}(n-1)$. Assume that, for some $k \in\left\{1, \ldots, k^{\prime}\right\}$, for every $i \in N,\left|u_{i}\left(\widetilde{x}^{k-1}\right)-u_{i}\left(x^{k-1}\right)\right| \leq \varepsilon^{\prime} \nu_{k-1}(n-1)$. We show that, for every $i \in N,\left|u_{i}\left(\widetilde{x}^{k}\right)-u_{i}\left(x^{k}\right)\right| \leq \varepsilon^{\prime} \nu_{k}(n-1)$. If $i \in W^{k}$, then $W^{k} \neq \emptyset$, and the statement follows from the observation that

$$
0 \leq u_{i}\left(\widetilde{x}^{k}\right)-u_{i}\left(x^{k}\right)=\varepsilon^{\prime} \nu_{k} \frac{\left|S^{k} \backslash W^{k}\right|}{\left|W^{k}\right|} \leq \varepsilon^{\prime} \nu_{k}(n-1) .
$$

If $i \in S^{k} \backslash W^{k}$, then we have that

$$
0 \geq u_{i}\left(\widetilde{x}^{k}\right)-u_{i}\left(x^{k}\right) \geq-\varepsilon^{\prime} \nu_{k} \geq-\varepsilon^{\prime} \nu_{k}(n-1) .
$$

If $i \in P^{k} \backslash S^{k}$, then $\left|u_{i}\left(\widetilde{x}^{k}\right)-u_{i}\left(x^{k}\right)\right|=0$. If $i \in N \backslash P^{k}$, then it holds that

$$
\left|u_{i}\left(\widetilde{x}^{k}\right)-u_{i}\left(x^{k}\right)\right|=\left|u_{i}\left(\widetilde{x}^{k-1}\right)-u_{i}\left(x^{k-1}\right)\right| \leq \varepsilon^{\prime} \nu_{k-1}(n-1)<\varepsilon^{\prime} \nu_{k}(n-1),
$$

where the first inequality makes use of the induction hypothesis and the last inequality of the fact that $\nu_{k-1}<\nu_{k}$.

Let some $k \in\left\{1, \ldots, k^{\prime}\right\}$ and some $i \in S^{k}$ be given. We show that $u_{i}\left(\widetilde{x}^{k}\right)>u_{i}\left(\widetilde{x}^{k-1}\right)$. If $i \in W^{k}$, then it holds that

$$
\begin{aligned}
u_{i}\left(\widetilde{x}^{k}\right) & =u_{i}\left(x^{k}\right)+\varepsilon^{\prime} \nu_{k} \frac{\left|S^{k} \backslash W^{k}\right|}{\left|W^{k}\right|} \\
& =u_{i}\left(x^{k-1}\right)+\varepsilon^{\prime} \nu_{k} \frac{\left|S^{k} \backslash W^{k}\right|}{\left|W^{k}\right|} \\
& \geq u_{i}\left(\tilde{x}^{k-1}\right)-\varepsilon^{\prime} \nu_{k-1}(n-1)+\varepsilon^{\prime} \nu_{k} \frac{1}{n-1} \\
& >u_{i}\left(\widetilde{x}^{k-1}\right)
\end{aligned}
$$


where the strict inequality uses that $\nu_{k}=n^{2} \nu_{k-1}$. If $i \in S^{k} \backslash W^{k}$, then it holds that

$$
\begin{aligned}
u_{i}\left(\widetilde{x}^{k}\right) & \geq u_{i}\left(x^{k}\right)-\varepsilon^{\prime} \nu_{k} \\
& \geq u_{i}\left(x^{k-1}\right)+\delta-\varepsilon^{\prime} \nu_{k} \\
& \geq u_{i}\left(\widetilde{x}^{k-1}\right)-\varepsilon^{\prime} \nu_{k-1}(n-1)+\delta-\varepsilon^{\prime} n^{2} \nu_{k-1} \\
& >u_{i}\left(\widetilde{x}^{k-1}\right)
\end{aligned}
$$

where the strict inequality uses the facts that $\delta \geq \varepsilon^{\prime}$ and

$$
\left(n^{2}+(n-1)\right) \nu_{k-1}<2 n^{2} \nu_{k-1} \leq 2 \nu_{k} \leq 1
$$

Combining the statements proven so far, it follows that $\widetilde{x}^{k^{\prime}} \in f^{k^{\prime}}\left(x^{0}\right)$. We complete the proof of the weak improvement property by noting that $x^{k^{\prime}} \in Y$ by the result of Béal, Rémila, and Solal (2013) and by demonstrating that $d\left(\widetilde{x}^{k^{\prime}}, x^{k^{\prime}}\right)<\varepsilon$. It follows that $d\left(\widetilde{x}^{k^{\prime}}, x^{k^{\prime}}\right)<\varepsilon$ since $\pi\left(\widetilde{x}^{k^{\prime}}\right)=\pi\left(x^{k^{\prime}}\right)$ and, for every $i \in N$,

$$
\left|u_{i}\left(\widetilde{x}^{k^{\prime}}\right)-u_{i}\left(x^{k^{\prime}}\right)\right| \leq \varepsilon^{\prime} \nu_{k^{\prime}}(n-1)<\varepsilon^{\prime} \leq \varepsilon .
$$

\subsection{One-to-One Matching}

As in Section 2.2, we can associate a social environment $\Gamma=\left(N,(X, d), E,\left(\succeq_{i}\right)_{i \in N}\right)$ to each one-to-one matching problem $(M, W, P)$. A matching $\mu$ is said to be stable in the matching problem $(M, W, P)$ if for every $i \in M \cup W$ it does not hold that $i P_{i} \mu(i)$ and if for every pair $(m, w) \in M \times W$ it does not hold that $w P_{m} \mu(m)$ and $m P_{w} \mu(w)$. It can easily be shown that a matching is stable if and only if it is in the core of the social environment $\Gamma$ as defined in Definition 4.13 .

In a seminal contribution to the literature, Gale and Shapley (1962) showed the existence of stable matchings. The following result of Roth and Vande Vate (1990) will also be helpful.

Lemma 5.6. (Roth and Vande Vate, 1990) For every matching $\mu \in X$ there is a stable matching $\mu^{\prime}$ such that $\mu^{\prime} \in f^{\mathbb{N}}(\mu)$.

Since the set of states is finite in this application, it holds that $f^{\mathbb{N}}(\mu)=f^{\infty}(\mu)$. As such, the result of Roth and Vande Vate (1990) can be rephrased as saying that $\Gamma$ satisfies the weak improvement property as defined in Definition 4.15. Given that for finite settings $f$ is always lower hemi-continuous, the following result now follows from Theorem 4.16.

Corollary 5.7. Let $(M, W, P)$ be a matching problem and let $\Gamma$ be the induced social environment as in Section 2.2. Then the MSS of $\Gamma$ is unique and equal to the set of stable matchings. 
Herings, Mauleon, and Vannetelbosch (2016) define the level-1 farsighted set for matching problems. It is not hard to see that the MSS for social environments $\Gamma$ as in Section 2.2 coincides with the level-1 farsighted set. Corollary 5.7 is therefore equivalent to Theorem 3 of Herings, Mauleon, and Vannetelbosch (2016) that characterizes the level-1 farsighted set as the core of the matching problem.

\subsection{Network Formation}

As in Section 2.3, we can associate a social environment $\Gamma=\left(N,(X, d), E,\left(\succeq_{i}\right)_{i \in N}\right)$ to each network problem $(N, \mathbb{G}, v)$. A network $g$ is said to be pairwise stable (Jackson and Wolinsky, 1996) if for every $i j \in g$ it holds that $v_{i}(g-i j) \leq v_{i}(g)$ and $v_{j}(g-i j) \leq v_{j}(g)$ and for every $i j \notin g$ it holds that $v_{i}(g+i j)>v_{i}(g)$ implies $v_{j}(g+i j) \leq v_{j}(g){ }^{2}$ It is not hard to show that a network is pairwise stable if and only if it is in the core of the social environment $\Gamma$ as defined in Definition 4.13.

Corollary 4.14 shows that any pairwise stable network is in the myopic stable set. However, it is not necessarily the case that the MSS only contains the pairwise stable networks.

Consider the binary relation $R$ on $X$ defined by $g R g^{\prime}$ if $g \in f^{\mathbb{N}}\left(g^{\prime}\right)$, i.e., $g$ can be reached from $g^{\prime}$ by a finite number of dominations. Let $I$ be the symmetric part of $R$, i.e., $g I g^{\prime}$ if and only if $g R g^{\prime}$ and $g^{\prime} R g$. Consider the set of equivalence classes $\mathbb{E}$ induced by $I$. Let us denote the equivalence class of network $g$ by $[g]$, i.e., $g^{\prime} \in[g]$ if and only if $g^{\prime} I g$. For two distinct equivalence classes $[g]$ and $\left[g^{\prime}\right]$ write $[g] P\left[g^{\prime}\right]$ if $g R g^{\prime}$. It is easy to see that $[g] P\left[g^{\prime}\right]$ if and only if $g R g^{\prime}$ and not $g R g^{\prime}$.

Let $V$ be the collection of maximal elements of $(\mathbb{E}, P)$, i.e., $[g] \in V$ if there is no $\left[g^{\prime}\right]$ such that $\left[g^{\prime}\right] P[g]$. Since an element of $V$ simply represents a closed cycle as defined in Definition 4.11, the following result follows from Theorem 4.12.

Corollary 5.8. Let $(N, \mathbb{G}, v)$ be a network problem and let $\Gamma$ be the induced social environment as in Section 2.3. A network $g$ belongs to an MSS $M$ if and only if the equivalence class $[g]$ belongs to $V$, i.e., $M=\{g \in X \mid[g] \in V\}$.

Herings, Mauleon, and Vannetelbosch (2009) define the pairwise myopically stable sets for network problems using the weaker notion of dominance corresponding to pairwise stability as defined in Jackson and Wolinsky (1996). It is not hard to see that the MSS for social environments $\Gamma$ as in Section 2.3 coincides with the pairwise myopically stable set for generic network problems. For such network problems, Corollary 5.8 is therefore equivalent

\footnotetext{
${ }^{2}$ Pairwise stability as defined in Jackson and Wolinsky (1996) is somewhat stronger and also requires that there is no $i j \notin g$ such that $v_{i}(g+i j)>v_{i}(g)$ and $v_{j}(g+i j)=v_{j}(g)$. The weaker notion used here is discussed as an alternative in Section 5 of Jackson and Wolinsky (1996) and is also widely used in the literature. For generic network problems, there are no indifferences, so the two definitions are equivalent.
} 
to Theorem 1 of Herings, Mauleon, and Vannetelbosch (2009) that characterizes the pairwise myopically stable set as the union of closed cycles. In their paper, a closed cycle is defined in the sense of Jackson and Watts (2002) for network problems. The notion of closed cycle of Definition 4.11 is the appropriate generalization to social environments.

\subsection{Normal-Form Games}

In Section 2.4, we associated a social environment $\Gamma=\left(N,(X, d), E,\left(\succeq_{i}\right)_{i \in N}\right)$ to each normalform game $G=\left(N,\left(\Sigma_{i}, d_{i}\right)_{i \in N},\left(\succeq_{i}\right)_{i \in N}\right)$.

A strategy profile $\sigma \in \Sigma$ is said to be a pure strategy Nash equilibrium of the game $G$ if, for every $i \in N$, for every $\sigma_{i}^{\prime} \in \Sigma_{i}$, it holds that $\sigma \succeq_{i}\left(\sigma_{i}^{\prime}, \sigma_{-i}\right)$. It can easily be shown that a strategy profile is a pure strategy Nash equilibrium if and only if it is in the core of the social environment $\Gamma$ as defined in Definition 4.13. Corollary 4.14 then shows that every pure strategy Nash equilibrium belongs to every MSS. For normal-form games, Theorem 4.16 reduces to the following result.

Corollary 5.9. Let $G$ be a normal-form game and let $\Gamma$ be the induced social environment as in Section 2.4. The MSS of $\Gamma$ is equal to the set of pure strategy Nash equilibria if and only if $\Gamma$ has the weak improvement property.

The following result exploits the fact that many classes of games have the weak improvement property.

Corollary 5.10. Let $G$ be a normal-form game and let $\Gamma$ be the induced social environment as in Section 2.4. Then the MSS is equal to the set of pure strategy Nash equilibria for finite potential games, aggregative games, and finite supermodular games.

Proof. For finite supermodular games, Friedman and Mezzetti (2001) show that the game has the weak finite improvement property which implies the weak improvement property. Monderer and Shapley (1996) establish the weak finite improvement property for potential games. For aggregative games, it is easily verified that $E$ is lower hemi-continuous and preferences are continuous by assumption, so $f$ is lower hemi-continuous by Theorem 4.9. Dindoš and Mezzetti (2006) show that aggregative games have the weak finite improvement property. The result now follows from Theorem 4.16 .

As an illustration, consider the two games in Example 4.

Example 4. Game 1 has a unique Nash equilibrium, but does not satisfy the weak finite improvement property. Thus, the MSS may contain strategy profiles which are not Nash equilibria. 


\section{Game 1:}

Player 2

\begin{tabular}{|c|c|c|c|c|}
\hline \multirow{5}{*}{$\begin{array}{ll} & A \\
\text { Player } 1 & B \\
& C \\
D\end{array}$} & $E$ & $F$ & $G$ & $H$ \\
\hline & 4,1 & $-20,-20$ & 1,4 & 0,0 \\
\hline & 2,2 & 4,1 & $-20,-20$ & 0,0 \\
\hline & $-20,-20$ & 2,2 & 4,1 & 0,0 \\
\hline & 0,0 & 0,0 & 0,0 & 1,1 \\
\hline
\end{tabular}

The Nash equilibrium of Game 1 is $(D, H)$ and the unique myopic stable set $M$ is given by

$$
M=\{(A, E),(A, G),(B, E),(B, F),(C, F),(C, G),(D, H)\}
$$

On the other hand, Game 2 shows that not every strategy profile where each strategy is played with positive probability in a mixed-strategy Nash equilibrium is part of an MSS. In this game there exists a pure strategy Nash equilibrium $(B, R)$ and two mixed strategy Nash equilibria

$$
\left(\left(\frac{1}{2} T, \frac{1}{2} M, 0 B\right), \quad\left(\frac{1}{2} L, \frac{1}{2} C, 0 R\right)\right),\left(\left(\frac{19}{42} T, \frac{1}{6} M, \frac{8}{21} B\right), \quad\left(\frac{8}{21} L, \frac{8}{21} C, \frac{5}{21} R\right)\right) .
$$

The unique MSS contains only the pure strategy Nash equilibrium $(B, R)$. Of course, if we define the states in the social environment corresponding to Game 2 to be the mixed strategy profiles, then the mixed Nash equilibria would be part of the MSS.

\section{Game 2:}

Player 2

\begin{tabular}{lc|c|c|c|} 
& \multicolumn{1}{c}{$L$} & \multicolumn{1}{c}{$C$} & \multicolumn{1}{c}{$R$} \\
\cline { 3 - 5 } Player 1 & $T$ & 1,3 & 3,1 & 0,0 \\
\cline { 3 - 5 } & $B$ & 3,1 & 1,3 & 0,0 \\
\cline { 3 - 5 } & $B$ & 0,0 & $\frac{3}{2}, \frac{3}{2}$ & 4,4 \\
\cline { 3 - 4 } & & & &
\end{tabular}

Asymmetric Bertrand Competition Theorem 4.1 shows that for any game $G$, the associated social environment $\Gamma$ has at least one MSS. Here, we illustrate the power of this result by determining the MSS in a two player Bertrand game with asymmetric costs. Consider two firms $N=\{1,2\}$ where each firm $i \in\{1,2\}$ chooses a price $p_{i} \geq 0$. We assume that the firm with the lowest price sells the amount $q \geq 0$ at its posted price and incurs a cost per unit of $c_{i} \geq 0$. In order to make the state space compact, take some $\bar{p}$ high enough, in particular exceeding $c_{1}$ and $c_{2}$, and restrict the prices to the interval $[0, \bar{p}]$. We consider the state space $X=\left\{p \in \mathbb{R}_{+}^{2} \mid p_{1} \leq \bar{p}, p_{2} \leq \bar{p}\right\}$ and the metric $d_{i}\left(p, p^{\prime}\right)=\left|p-p^{\prime}\right|, i=1,2$. 
Additionally, we assume that costs are asymmetric, so $c_{1}<c_{2} \cdot{ }^{3}$ If both firms post the same price, output is split equally between the two firms. Profits (and therefore preferences) are given by

$$
u_{i}\left(p_{i}, p_{j}\right)= \begin{cases}\left(p_{i}-c_{i}\right) q, & \text { if } p_{i}<p_{j} \\ \left(p_{i}-c_{i}\right) q / 2, & \text { if } p_{i}=p_{j} \\ 0, & \text { if } p_{i}>p_{j}\end{cases}
$$

This game is interesting for several reasons. To start, observe that the payoff functions are not continuous. Given this, the dominance correspondence $f$ is not lower hemi-continuous, so we cannot use Theorem 4.6 to establish uniqueness of the MSS. Also, apart from the discontinuity in payoff functions, this asymmetric Bertrand game has no pure strategy Nash equilibria, so the core of the associated social environment is empty. Finally, several papers mistakenly claim that this game has no Nash equilibrium. As Blume (2003) noted, there are mixed Nash equilibria in which player 1 chooses $p_{1}=c_{2}$ and player 2 randomizes his prize $p_{2}$ continuously on an interval $\left[c_{2}, c_{2}+\varepsilon\right]$. On the other hand, the literature has so far not yet been able to determine all mixed Nash equilibria of this game.

To illustrate the versatility of our framework, let us determine an MSS of this game and show it is unique. It is represented by the shaded area in Figure 1. The construction, which crucially relies on the fact that the MSS is closed, proceeds in several steps. Let $M$ be an MSS, which exists by virtue of Theorem 4.1.

Step 1. $P^{1}=\left\{\left(p_{1}, p_{2}\right) \in X \mid c_{1} \leq p_{1}=p_{2} \leq c_{2}\right\} \subseteq M$.

Towards a contradiction, suppose that $\left(p_{1}, p_{2}\right) \in X$ satisfies $c_{1}<p_{1}=p_{2}<c_{2}$ and $\left(p_{1}, p_{2}\right)$ is not in $M$. Once the contradiction is obtained, we get the result of Step 1 exploiting the fact that $M$ is closed.

Take any $p_{1}^{\prime} \in \mathbb{R}_{+}$such that $c_{1}<p_{1}^{\prime}<p_{1}$. There are two cases to consider. In case $1,\left(p_{1}^{\prime}, p_{2}\right) \in M$. Given that $\left(p_{1}, p_{2}\right) \notin M$ and $M$ is closed, there is an $\varepsilon^{\prime}>0$ such that for every $\varepsilon \in\left(0, \varepsilon^{\prime}\right)$ we have $\left(p_{1}-\varepsilon, p_{2}\right) \notin M$. However, for $\varepsilon$ small enough, $\left(p_{1}-\varepsilon, p_{2}\right) \in f\left(p_{1}^{\prime}, p_{2}\right)$ as firm 1 will find it profitable to deviate to $p_{1}-\varepsilon>p_{1}^{\prime}$. Since $M$ satisfies deterrence of external deviations, it follows that $\left(p_{1}-\varepsilon, p_{2}\right) \in M$, leading to a contradiction. In case 2 , we have $\left(p_{1}^{\prime}, p_{2}\right) \notin M$. By external stability, there must be $\left(p_{1}^{\prime \prime}, p_{2}^{\prime \prime}\right) \in M$ such that $\left(p_{1}^{\prime \prime}, p_{2}^{\prime \prime}\right) \in f^{\infty}\left(p_{1}^{\prime}, p_{2}\right)$. At $\left(p_{1}^{\prime}, p_{2}\right)$, firm 2 makes no sales and has zero profits. Since $p_{1}^{\prime}<c_{2}$, it has no profitable deviation. For firm 1 , any $\tilde{p}_{1} \in \mathbb{R}_{+}$such that $p_{1}^{\prime}<\tilde{p}_{1}<p_{2}$ is a profitable deviation, $\tilde{p}_{1}=p_{2}$ may or may not be a profitable deviation, and $\tilde{p}_{1}>p_{2}$ is not a profitable deviation. It is now easy to see that $f^{\infty}\left(p_{1}^{\prime}, p_{2}\right)=\left\{\left(\tilde{p}_{1}, p_{2}\right) \in \mathbb{R}_{+}^{2} \mid p_{1}^{\prime} \leq \tilde{p}_{1} \leq p_{2}\right\}$. External stability therefore implies

\footnotetext{
${ }^{3}$ In the symmetric case $c_{1}=c_{2}=c$, it is easily verified that the unique MSS is equal to the unique Nash equilibrium, i.e., $p_{1}=p_{2}=c$.
} 
that there is $\left(p_{1}^{\prime \prime}, p_{2}\right) \in M$ with $c_{1}<p_{1}^{\prime \prime}<p_{1}$, but then we are back in case 1 , and we obtain a contradiction as before. Consequently, it holds that $\left(p_{1}, p_{2}\right) \in M$.

Step 2. $P^{2}=\left\{\left(p_{1}, p_{2}\right) \in X \mid c_{1} \leq p_{1} \leq c_{2}, p_{1} \leq p_{2}\right\} \subseteq M$.

Take $\left(p_{1}, p_{2}\right) \in P^{1}$ such that $p_{2}<c_{2}$. It follows from Step 1 that $\left(p_{1}, p_{2}\right) \in M$. It holds that $u_{2}\left(p_{1}, p_{2}\right)$ is strictly negative. As such, firm two can gain by increasing $p_{2}$ above the value of $p_{1}$ as this will give him a profit of zero. By deterrence of external deviations, all these options must also be in $M$. The result of Step 2 now follows from the requirement that $M$ is closed.

Step 3. $P^{3}=\left\{\left(p_{1}, p_{2}\right) \in X \mid c_{1} \leq p_{1} \leq p_{2}\right\} \subseteq M$.

Take $\left(p_{1}, p_{2}\right) \in P^{2}$ such that $c_{1}<p_{1}<p_{2}$. By Step 2 it holds that $\left(p_{1}, p_{2}\right) \in M$. Then firm 1 can deviate and can increase profits by choosing $p_{1}^{\prime}$ such that, $p_{1}<p_{1}^{\prime}<p_{2}$. Since $M$ satisfies deterrence of external deviations, it holds that $\left(p_{1}^{\prime}, p_{2}\right) \in M$. This shows that we can drop the restriction $p_{1} \leq c_{2}$ from the definition of the set $P^{2}$. Using closedness of $M$ we can again change strict inequalities to weak inequalities.

Step 4. $P^{4}=P^{3} \cup\left\{\left(p_{1}, p_{2}\right) \in X \mid c_{2} \leq p_{2} \leq p_{1}\right\} \subseteq M$.

Take $\left(p_{1}, p_{2}\right) \in P^{3}$ such that $c_{2}<p_{1}<p_{2}$. By Step 3 it holds that $\left(p_{1}, p_{2}\right) \in M$. Now firm 2 can deviate and set $p_{2}^{\prime}$ such that $c_{2}<p_{2}^{\prime}<p_{1}$ and make strictly positive profits. Thus, the set $\left\{\left(p_{1}, p_{2}\right) \in X \mid c_{2} \leq p_{2} \leq p_{1}\right\}$ is a subset of $M$. The set $P^{4}$ is given by the shaded area in the Figure 1.

Step 5. $P^{4}$ is the unique MSS.

We have shown that $P^{4}$ is contained in any MSS, so we only need to show that $P^{4}$ itself is a QMSS. First, observe that $P^{4}$ is closed. Next, $X \backslash P^{4}$ is given by

$$
\underbrace{\left\{\left(p_{1}, p_{2}\right) \in X \mid p_{1}<c_{1}\right\}}_{P^{5}} \cup \underbrace{\left\{\left(p_{1}, p_{2}\right) \in X \mid p_{1}>p_{2}, p_{2}<c_{2}\right\}}_{P^{6}} .
$$

In order to see that $P^{4}$ satisfies deterrence of external deviations, observe that firm 1 will never deviate to a point in the set $P^{5}$ as this gives zero or negative profits for firm 1 and profits at states in $P^{4}$ are non-negative for firm 1. Firm 2 has no possibility to deviate to $P^{5}$ from a point in the set $P^{4}$. Also, any point in the set $P^{6}$ gives firm 2 negative profits. Firm 2 only obtains negative profits at states in $P^{4}$ if $p_{1}=p_{2}<c_{2}$. However, if firm 2 deviates to $p_{2}^{\prime}<p_{1}$, then his profits would become more negative so firm 2 will never deviate to states in $P^{6}$.

It remains to show that $P^{4}$ satisfies external stability. If $p_{2} \leq p_{1}<c_{1}$, then firm 2 can gain by choosing $p_{2}^{\prime}$ such that $p_{2}^{\prime}>c_{2}$. Next firm 1 can gain by choosing $p_{1}^{\prime}$ such that $c_{2}<p_{1}^{\prime}<p_{2}$. The strategy profile $\left(p_{1}^{\prime}, p_{2}^{\prime}\right)$ belongs to $P^{4}$. If $p_{1}<p_{2} \leq c_{1}$, then firm 1 
Figure 1: The MSS for the asymmetric Bertrand model. Left panel: without legal restrictions. Right panel: with legal restrictions.
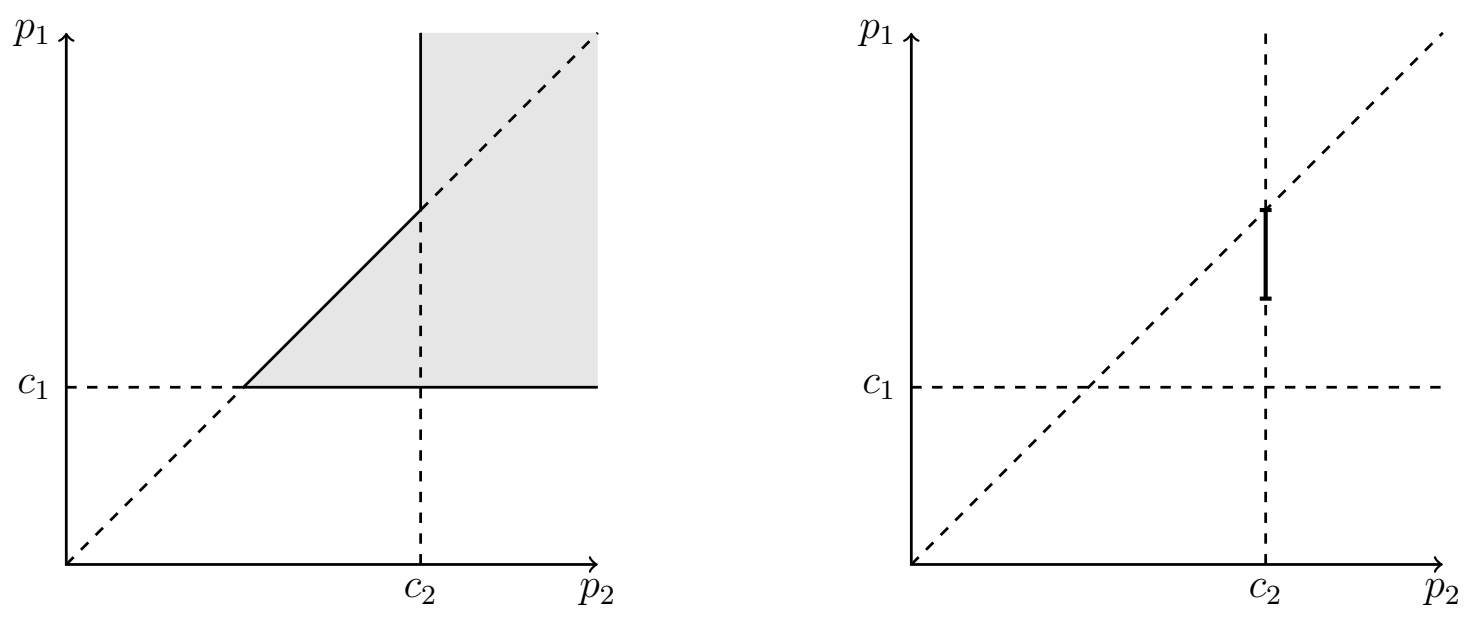

can gain by choosing $p_{1}^{\prime}$ such that $p_{1}^{\prime}>c_{2}$. Then firm 2 can gain by choosing $p_{2}^{\prime}$ such that $c_{2}<p_{2}^{\prime}<p_{1}^{\prime}$. The strategy profile $\left(p_{1}^{\prime}, p_{2}^{\prime}\right)$ is in $P^{4}$. If $p_{1}<c_{1}<p_{2}$, firm 1 can gain by choosing $p_{1}^{\prime}$ such that $c_{1}<p_{1}^{\prime}<p_{2}$ which leads to the strategy profile $\left(p_{1}^{\prime}, p_{2}\right)$ in $P^{4}$. Hence, external stability holds starting from any state in $P^{5}$. For $\left(p_{1}, p_{2}\right) \in P^{6} \backslash P^{5}$ it holds that $p_{1}>p_{2}, c_{1} \leq p_{1}$, and $p_{2}<c_{2}$, so firm 2 can gain by choosing $p_{2}^{\prime}=c_{2}$. The strategy profile $\left(p_{1}, c_{2}\right)$ belongs to $P^{4}$.

Asymmetric Bertrand Competition under Legal Restrictions Let us now consider a slightly different version of Bertrand competition. In many countries, pricing below marginal or average cost is considered to be predatory pricing and thus forbidden by law. We analyze how this restriction influences the MSS. To do so, we adjust the state space and define

$$
X=\left\{\left(p_{1}, p_{2}\right) \in P \mid c_{1} \leq p_{1}, c_{2} \leq p_{2}\right\}
$$

The MSS is considerably smaller than in the previous setting. In particular, we will show that it is equal to the set

$$
P^{*}=\left\{\left(p_{1}, c_{2}\right) \in P \mid \frac{c_{1}+c_{2}}{2} \leq p_{1} \leq c_{2}\right\} .
$$

Recall that the mixed Nash equilibrium derived by Blume (2003) had $p_{1}=c_{2}$ and $p_{2}$ being drawn from an atomless distribution on an interval $\left[c_{2}, c_{2}+\varepsilon\right]$. The prediction of the MSS is that prices will be set lower than in the mixed Nash equilibrium.

Again, we split the proof into several steps. 
Step 1. First we show that $P^{*}$ is a QMSS. We first establish deterrence of external deviations. For $\left(p_{1}, c_{2}\right) \in P^{*}$, profits of firm 1 are non-negative. Thus, setting $p_{1}>c_{2}$ with a payoff of zero is not a profitable deviation for firm 1 from any point in $P^{*}$. Note that the payoff of player 1 is increasing in $p_{1}$ for $p_{1}<c_{2}$. Thus, a deviation to a $p_{1}<\left(c_{1}+c_{2}\right) / 2$ could only be profitable from the strategy profile $\left(c_{2}, c_{2}\right)$. This requires $\left(p_{1}-c_{1}\right) q>\left(c_{2}-c_{1}\right) q / 2$ or, equivalently, $p_{1}>\left(c_{1}+c_{2}\right) / 2$, which is not the case. It is easily verified that firm 2 cannot increase its profits by deviating from any $\left(p_{1}, c_{2}\right) \in P^{*}$. This shows deterrence of external deviations for $P^{*}$.

It remains to verify external stability. Let some state $\left(p_{1}, p_{2}\right) \in X \backslash P^{*}$ be given. If $c_{2}<p_{1}<p_{2}$, then firm 2 can profitably deviate to $p_{2}^{\prime}=\left(c_{2}+p_{1}\right) / 2$ and firm 1 can profitably deviate in the next step to $p_{1}^{\prime}=\left(c_{2}+p_{2}^{\prime}\right) / 2$ and so forth. It follows that $\left(c_{2}, c_{2}\right) \in f^{\infty}\left(p_{1}, p_{2}\right)$. If $p_{1} \leq c_{2}<p_{2}$, then firm 1 can profitably deviate to $p_{1}^{\prime}$ such that $c_{2}<p_{1}^{\prime}<p_{2}$ and we can continue as in the previous case. If $c_{2}<p_{2} \leq p_{1}$, then firm 1 can profitably deviate to $p_{1}^{\prime}$ such that $c_{2}<p_{1}^{\prime}<p_{2}$ and we can continue as before. If $p_{1} \notin\left[\left(c_{1}+c_{2}\right) / 2, c_{2}\right]$ and $p_{2}=c_{2}$, then firm 1 can profitably deviate to $p_{1}^{\prime}=\left(c_{1}+c_{2}\right) / 2$ to reach a state in $P^{*}$. We have covered all states in $X \backslash P^{*}$ and thereby shown that $P^{*}$ satisfies external stability.

Step 2. Let $M$ be a QMSS. Let us show that for every $\left(p_{1}, c_{2}\right) \in P^{*} \backslash\left\{\left(c_{2}, c_{2}\right)\right\}$, if $\left(p_{1}, c_{2}\right) \in M$, then $\left(c_{2}, c_{2}\right) \in M$. Suppose $\left(c_{2}, c_{2}\right) \notin M$. By closedness of $M$, there is $\bar{\varepsilon}>0$ such that, for every $\varepsilon \in(0, \bar{\varepsilon}),\left(c_{2}-\varepsilon, c_{2}\right) \notin M$. Take $p_{1}^{\prime}=\max \left\{\left(p_{1}+c_{2}\right) / 2, c_{2}-\bar{\varepsilon} / 2\right\}$, then $\left(p_{1}^{\prime}, c_{2}\right) \in f\left(p_{1}, c_{2}\right)$, so $\left(p_{1}^{\prime}, c_{2}\right) \in M$. Given that $p_{1}^{\prime}>c_{2}-\bar{\varepsilon}$, we obtain a contradiction.

Step 3. Let $M$ be a QMSS. Let us show that if $\left(c_{2}, c_{2}\right) \in M$, then, for every $\left(p_{1}, c_{2}\right) \in P^{*} \backslash$ $\left\{\left(c_{2}, c_{2}\right)\right\}$, we have $\left(p_{1}, c_{2}\right) \in M$. This follows from the fact that any strategy profile in $\left(p_{1}, c_{2}\right) \in P^{*} \backslash\left\{\left(c_{2}, c_{2}\right)\right\}$ with $p_{1}>\left(c_{1}+c_{2}\right) / 2$ offers higher profits for firm 1 compared to $\left(c_{2}, c_{2}\right)$ and the fact that $M$ is closed.

Step 4. We are now ready to show that $P^{*}$ is an MSS. First of all, by step 1 it is a QMSS. So if, towards a contradiction, $P^{*}$ is not an MSS, it should violate minimality. This means that there is a proper subset of $P^{*}$ that is also a QMSS. This subset either contains $\left(c_{2}, c_{2}\right)$ or it is a subset of $P^{*} \backslash\left\{\left(c_{2}, c_{2}\right)\right\}$. If contains $\left(c_{2}, c_{2}\right)$ then, by Step 3, it should contain $P^{*} \backslash\left\{\left(c_{2}, c_{2}\right)\right\}$ and therefore be equal to $P^{*}$. If it is a subset of $P^{*} \backslash\left\{\left(c_{2}, c_{2}\right)\right\}$, then by Step 2 , it should contain $\left(c_{2}, c_{2}\right)$, a contradiction.

Step 5. Finally, let us show that the set $P^{*}$ is the unique MSS. Let $M$ be an MSS. By Lemma 4.3 , it holds that $P^{*} \cap M \neq \emptyset$. If $M$ contains $\left(c_{2}, c_{2}\right)$, then, by Step $3, M$ should also contain $P^{*} \backslash\left\{\left(c_{2}, c_{2}\right)\right\}$, so $P^{*} \subseteq M$ and by minimality $P^{*}=M$. If $M$ contains an 
element of $P^{*} \backslash\left\{\left(c_{2}, c_{2}\right)\right\}$, then, by Step 2, it should also contain $\left(c_{2}, c_{2}\right)$ and, by Step

3, also $P^{*} \backslash\left\{\left(c_{2}, c_{2}\right)\right\}$. Again, we obtain $P^{*} \subseteq M$ and by minimality $P^{*}=M$.

By characterizing the MSS for the asymmetric Bertrand model, we have shown that it is possible to find the MSS in nontrivial non-cooperative games. The difference between the MSS in the two versions of the Bertrand model given above is substantial which emphasizes the great importance of details in this model, i.e., the choice of strategy sets. The underlying origin of this sensitivity is due to the discontinuity in payoff functions. Note that in both cases no pure strategy Nash equilibrium exists, any mixed-strategy Nash equilibrium involves randomizations over a continuous interval, and the literature contains no full characterization of the set of Nash equilibria. The fact that it is not overly complicated to characterize the MSS in such a complex environment further boosts the appeal of the MSS as an equilibrium concept.

\section{Conclusion}

The myopic stable set provides a solution concept for a wide variety of social environments. As we have shown, the setting encompasses coalition function form games, models of network formation, matching models, and non-cooperative games. These environments have been chosen based on their prominence in the literature but are by no means exhaustive. In particular, promising environments for future research on the myopic stable set include exchange processes in general equilibrium models, many-to-many matching with transfers and non-cooperative games where groups of players can deviate.

The following three features boost the appeal of the myopic stable set as a solution concept. First, the myopic stable set unifies standard solution concepts in many social environments. For instance, it coincides with the coalition structure core in coalition function form games (Kóczy and Lauwers, 2004) if the coalition structure core is non-empty, the set of stable matchings in the standard one-to-one matching model (Gale and Shapley, 1962), the set consisting of pairwise stable networks and closed cycles of networks (Jackson and Watts, 2002), and the set of pure strategy Nash equilibria in finite supermodular games (Bulow, Geanakoplos, and Klemperer, 1985), finite potential games (Monderer and Shapley, 1996), and aggregative games (Selten, 1970).

Second, our solution concept exists for any social environment and-under weak continuity assumptions - provides a unique (set-valued) prediction. This differs from well-known concepts in the literature which fail to satisfy these properties even in social environments with more structure.

Finally, we have characterized the myopic stable set explicitly for two versions of asymmetric Bertrand competition, a complex game which contains no pure strategy Nash equi- 
librium and where the set of mixed strategy Nash equilibria is unknown. Thus, we believe that the myopic stable set can be fruitfully applied in many settings.

\section{References}

Aliprantis, C.D., And K.C. Border (2006), Infinite Dimensional Analysis - A Hitchhiker's Guide, Springer, Berlin.

Aumann, R.J. (1959), "Acceptable Points in General Cooperative n-Person Games," in A.W. Tucker and R.D. Luce (eds.), Contributions to the Theory of Games, Volume IV, Princeton University Press, Princeton, pp. 287-324.

Bala, V., And S. Goyal (2000), "A Noncooperative Model of Network Formation," Econometrica, 68, 1181-1229.

BÉAl, S., E. RÉmila, And P. Solal (2013), "Accessibility and Stability of the Coalition Structure Core," Mathematical Methods of Operations Research, 78, 187-202.

Blume, A. (2003), "Bertrand without Fudge," Economics Letters, 78, 167-168.

Bondareva, O.N. (1963), "Some Applications of Linear Programming Methods to the Theory of Cooperative Games," Problemy Kibernetiki, 10, 119-139.

Bulow, J.I., J.D. Geanakoplos, And P.D. Klemperer (1985), "Multimarket Oligopoly: Strategic Substitutes and Complements," Journal of Political Economy, 93, 488-511.

Chwe, M. S.-Y. (1994), "Farsighted Coalitional Stability," Journal of Economic Theory, 63, 299-325.

Dindoš, M., And C. Mezzetti (2006), "Better-reply Dynamics and Global Convergence to Nash Equilibrium in Aggregative Games," Games and Economic Behavior, 54, 261292 .

Dutta, B., S. Ghosal, And D. RAY (2005), "Farsighted Network Formation," Journal of Economic Theory, 122, 143-164.

Dutta, B., And S. Mutuswami (1997), "Stable Networks," Journal of Economic Theory, $76,322-344$.

Friedman, J.W., And C. Mezzetti (2001), "Learning in Games by Random Sampling," Journal of Economic Theory, 98, 55-84. 
Gale, D., And L.S. Shapley (1962), "College Admissions and the Stability of Marriage," American Mathematical Monthly, 69, 9-15.

Hart, S., And M. Kurz (1983), "Endogenous Formation of Coalitions," Econometrica, $51,1047-1064$.

Herings, P.J.J., A. Mauleon, And V. Vannetelbosch (2004), "Rationalizability for Social Environments," Games and Economic Behavior, 49 135-156.

Herings, P.J.J., A. Mauleon, And V. Vannetelbosch (2009), "Farsightedly Stable Networks," Games and Economic Behavior, 67, 526-541.

Herings, P.J.J., A. Mauleon, And V. Vannetelbosch (2014), "Stability of Networks under Level- $K$ Farsightedness," METEOR Research Memorandum 14/30, Maastricht University, Maastricht, pp. 1-39.

Herings, P.J.J., A. Mauleon, And V. Vannetelbosch (2016), "Stable Sets in Matching Problems with Coalitional Sovereignty and Path Dominance," GSBE Research Memorandum 16/20, Maastricht University, Maastricht, pp. 1-15.

Jackson, M.O., And A. VAn Den Nouweland (2005), "Strongly Stable Networks," Games and Economic Behavior, 51, 420-444.

JACKSOn, M.O., AND A. WATts (2002), "The Evolution of Social and Economic Networks," Journal of Economic Theory, 106, 265-295.

Jackson, M.O., And A. Wolinsky (1996), "A Strategic Model of Social and Economic Networks," Journal of Economic Theory, 71, 44-74.

Kóczy, L.Á., AND L. LAuwers (2004), "The Coalition Structure Core Is Accessible," Games and Economic Behavior, 48, 86-93.

Konishi, H., And D. RAY (2003), "Coalition Formation as a Dynamic Process," Journal of Economic Theory, 110, 1-41.

Lucas, W.F. (1968), "A Game with no Solution," Bulletin of the American Mathematical Society, 74, 237-239.

LuCAs, W.F. (1992), "Von Neumann-Morgenstern Stable Sets," in R.J. Aumann and S. Hart (eds.), Handbook of Game Theory, Volume 1, Elsevier Science Publishers, Amsterdam, pp. 543-590.

Monderer, D., And L.S. Shapley (1996), "Potential Games," Games and Economic Behavior, 14, 124-143. 
Page, F.H., JR., And M. Wooders (2009), "Strategic Basins of Attraction, the Path Dominance Core, and Network Formation Games," Games and Economic Behavior, $66,462-487$.

Page, F.H., Jr., M. Wooders, and S. Kamat (2005), "Networks and Farsighted Stability," Journal of Economic Theory, 120, 257-269.

Ray, D., And R. Vohra (2014), "Coalition Formation," in H.P. Young and S. Zamir (eds.), Handbook of Game Theory, Volume 4, Elsevier, Amsterdam, pp. 239-326.

Ray, D., And R. Vohra (2015), "The Farsighted Stable Set," Econometrica, 83, 9771011.

Roth, A.E., And J.H. Vande Vate (1990), "Random Paths to Stability in Two-sided Matching," Econometrica, 58, 1475-1480.

ScArf, H. (1967), "The Core of an N Person Game," Econometrica, 35, 50-69.

Selten, R. (1970), Preispolitik der Mehrproduktenunternehmung in der statischen Theorie, Springer-Verlag, Berlin.

Shapley, L.S. (1967), "On Balanced Sets and Cores," Naval Research Logistics Quarterly, 14, 453-460.

Shubik, M. (1962), Game Theory in the Social Sciences: Concepts and Solutions, MIT Press, Cambridge, Massachusetts.

Xue, L. (1998), "Coalitional Stability under Perfect Foresight," Economic Theory, 11, 603-627. 